\title{
EQUIVARIANT AND INVARIANT THEORY OF NETS OF CONICS WITH AN APPLICATION TO THOM POLYNOMIALS
}

\author{
M. DOMOKOS, L. M. FEHÉR, AND R. RIMÁNYI
}

\begin{abstract}
Two parameter families of plane conics are called nets of conics. There is a natural group action on the vector space of nets of conics, namely the product of the group reparametrizing the underlying plane, and the group reparametrizing the parameter space of the family. We calculate equivariant fundamental classes of orbit closures. Based on this calculation we develop the invariant theory of nets of conics. As an application we determine Thom polynomials of contact singularities of type $(3,3)$. We also show how enumerative problems, in particular the intersection multiplicities of the determinant map from nets of conics to plane cubics, can be solved studying equivariant classes of orbit closures.
\end{abstract}

\section{INTRODUCTION}

The Thom polynomial is a multivariable polynomial associated with a singularity, governing its global behavior. Thom polynomials of certain singularities are known, mostly of those with small codimension, or with some other simple structure. The Thom polynomials of the simplest so-called $\Sigma^{3}$ singularities are not known. The first objective of this paper is to calculate the Thom polynomials of these simplest $\Sigma^{3}$ singularities. These singularities are closely related with nets (i.e., two-parameter families) of (plane) conics. Recent work of the second and the third authors [FR12] reduces the calculations of these Thom polynomials to the calculation of the equivariant cohomology classes of the corresponding classes of nets of conics.

While calculating the equivariant cohomology classes of nets of conics we realized that most of the classical results on the invariant theory of nets of conics can be reproduced using our equivariant techniques. We included the invariant theory of nets of conics and its relation with the equivariant theory for two reasons. First, this makes the paper more self-contained, and second, we find the relation between equivariant and invariant theory interesting, and possibly applicable in the future for more general representations.

Another byproduct of our calculations is a list of intersection multiplicities for the determinant map (assigning a plane cubic to a net of conics, its discriminant). Intersection multiplicities are difficult to calculate in general. We will define and compute equivariant intersection multiplicities, and show that they agree with the non-equivariant ones.

\section{Structure of the PAPER}

Two parameter families of plane conics are called nets of conics. There is a natural group action on the vector space of nets of conics, namely the product of the group of linear reparametrizations of the underlying plane, and the group of linear reparametrizations of the parameter space of the family.

In Section 3 we develop the equivariant cohomology theory of nets of conics with respect to this action. The results of the section are summarized in Theorems 3.1 and 3.3.

The first named author is supported by OTKA grants NK 81203 and K 101515 . The second author is supported by the OTKA grants 72537 and 81203. The third author is supported by NSA grant CON:H98230-10-1-0171. 
M. DOMOKOS, L. M. FEHÉR, AND R. RIMÁNYI

In Section 4, using results of [FR12], we determine Thom polynomials of contact singularities of type $(3,3)$. The main results of the paper are Theorem 4.1 and 4.4 .

In Section 5 we develop the invariant theory of nets of conics. While this theory is mostly known, we emphasize that the equivariant point of view gives a conceptional way to approach invariant theory. This section however is relatively independent of the rest of the paper, it does not use cohomology theory. It is known that the corresponding ring of invariants is generated by two algebraically independent invariants having degree 6 and 12, see Section 5 for references. We give a formula for the degree 6 invariant (already known to Salmon and Sylvester) in terms of the Plücker coordinates as a pull-back of a degree 2 invariant (6) of the Plücker space. The expression (6) for this degree 2 invariant appears to be new.

In Section 6 we describe the hierarchy (see Figure 2) of the orbits of nets of conics. This hierarchy was first obtained by different techniques in [Wal77]. We show how the cohomological data obtained in Section 3 through the notion of incidence class can recover the result on hierarchy. This method of reducing hierarchy to equivariant cohomology calculations may be applied in the future to other representations with small GIT quotients. The technique is particularly promising in the emerging field of Geometric Complexity Theory, see [Mul09].

In Section 7 we show how enumerative problems, in particular the intersection multiplicities of the determinant map from nets of conics to plane cubics, can be solved studying the equivariant cohomology classes of the orbits. The main result of the section is Theorem 7.1. We also explain that in some cases the intersection multiplicities agree with the algebraic multiplicities. We hope that these calculations may lead to the determination of which orbit-closures of the plane cubics are Cohen-Macaulay (see Remark 7.2). For completeness we included the multiplicities of the induced map between the corresponding GIT quotients though these results are probably known.

Throughout the paper we work in the complex algebraic category, hence in particular, GL $(U)$ means the group of complex linear transformations of the complex vector space $U$, and $\mathrm{GL}_{n}$ denotes $\mathrm{GL}\left(\mathbb{C}^{n}\right)$. Cohomology will be considered with integer coefficients.

The authors are grateful to M. Kazarian for several useful discussions on Thom polynomials and to C. T. C. Wall for very valuable comments on nets of conics. Additionally the authors would like to thank I. Dolgachev, J. Chipalkatti, L. Oeding and P. Frenkel for useful conversations on the topics in this paper.

\section{Classification of ORBItS OF NETS AND THEIR EQUivaRIant ClasseS}

3.1. Orbits of nets of conics. Let $S^{2} U$ denote the second symmetric power of the vector space (or representation) $U$. Consider the vector spaces $U=\mathbb{C}^{3}$ and $V=\mathbb{C}^{3}$. The main object of this paper is the $\mathrm{GL}(U) \times \mathrm{GL}(V)$ representation $\mathbf{N o c}=\operatorname{Hom}\left(S^{2} U, V\right)$.

Through the natural isomorphism $\operatorname{Hom}\left(S^{2} U, V\right)=\operatorname{Hom}\left(V^{*}, S^{2} U^{*}\right)$, elements of this vector space are families of homogeneous degree 2 polynomials on $U$ parameterized by the vector space $V^{*}$. Lines in $S^{2} U^{*}$ determine conics in the projective plane $\mathbf{P}(U)$, hence elements of $\operatorname{Hom}\left(V^{*}, S^{2} U^{*}\right)$ are 2-parameter families (nets) of plane conics (Noc stands for nets of con$i c s)$. The $\mathrm{GL}(U)$ action reparametrizes the underlying plane $\mathbf{P}(U)$, and the $\mathrm{GL}(V)$ action reparametrizes the parameter space $V^{*}$.

There is a natural stratification $\Sigma^{2} \cup \Sigma^{1} \cup \Sigma^{0}$ of Noc $-\{0\}$, according to corank. Geometrically the strata correspond to conics, pencils of conics (i.e., 1-parameter families of conics), and (proper) nets of conics, respectively. The orbit structure of conics and pencils of conics is widely known. The classification of orbits of proper nets of conics is given in [Wal77] for the codimension $>1$ cases and in [WdP95] for the family of codimension 1 orbits. We will use their notations. 
TABLE 1. Codimension> 1 orbits and symmetries

\begin{tabular}{|c|c|c|c|c|c|}
\hline & $\mathrm{cd}$ & representative & symmetry & Poincaré & $\delta$ \\
\hline \multicolumn{6}{|l|}{$\Sigma^{0}$} \\
\hline$C$ & 2 & $y^{2}+2 x z, 2 y z,-x^{2}$ & $(2 \alpha, \alpha+\beta, 2 \beta),(2 \alpha+2 \beta, \alpha+3 \beta, 4 \alpha)$ & 1,1 & $\nu$ \\
\hline$D$ & 2 & $x^{2}, y^{2}, z^{2}+2 x y$ & $(2 \alpha, 2 \beta, \alpha+\beta),(4 \alpha, 4 \beta, 2 \alpha+2 \beta)$ & 1,2 & $\theta$ \\
\hline$D^{*}$ & 2 & $2 x z, 2 y z, z^{2}+2 x y$ & $(2 \alpha, 2 \beta, \alpha+\beta),(3 \alpha+\beta, \alpha+3 \beta, 2 \alpha+2 \beta)$ & 1,2 & $\theta$ \\
\hline$E$ & 3 & $x^{2}, y^{2}, z^{2}$ & $(\alpha, \beta, \gamma),(2 \alpha, 2 \beta, 2 \gamma)$ & $1,2,3$ & A \\
\hline$E^{*}$ & 3 & $2 x y, 2 y z, 2 z x$ & $(\alpha, \beta, \gamma),(\alpha+\beta, \beta+\gamma, \gamma+\alpha)$ & $1,2,3$ & A \\
\hline$F$ & 3 & $x^{2}+y^{2}, 2 x y, 2 y z$ & $(\alpha, \alpha, \beta),(2 \alpha, 2 \alpha, \alpha+\beta)$ & 1,1 & $\neq$ \\
\hline$F^{*}$ & 3 & $x^{2}+y^{2}, x z, z^{2}$ & $(\alpha, \alpha, \beta),(2 \alpha, \alpha+\beta, 2 \beta)$ & 1,1 & $\Omega$ \\
\hline$G$ & 4 & $x^{2}, y^{2}, y z$ & $(\alpha, \beta, \gamma),(2 \alpha, 2 \beta, \beta+\gamma)$ & $1,1,1$ & $\neq$ \\
\hline$G^{*}$ & 4 & $x y, x z, z^{2}$ & $(\alpha, \beta, \gamma),(\alpha+\beta, \alpha+\gamma, 2 \gamma)$ & $1,1,1$ & $\neq$ \\
\hline$H$ & 5 & $x^{2}, 2 x y, y^{2}+2 x z$ & $(2 \alpha, \alpha+\beta, 2 \beta),(4 \alpha, 3 \alpha+\beta, 2 \alpha+2 \beta)$ & 1,1 & $\Xi$ \\
\hline$I$ & 7 & $x^{2}, x y, y^{2}$ & $(\alpha, \beta, \gamma),(2 \alpha, \alpha+\beta, 2 \beta)$ & $1,1,2$ & 0 \\
\hline$I^{*}$ & 7 & $x z, y z, z^{2}$ & $(\alpha, \beta, \gamma),(\alpha+\gamma, \beta+\gamma, 2 \gamma)$ & $1,1,2$ & 0 \\
\hline \multicolumn{6}{|l|}{$\Sigma^{1}$} \\
\hline$\left(1^{4}\right)$ & 4 & $x^{2}-x z, y^{2}-y z, 0$ & $(\alpha, \alpha, \alpha),(2 \alpha, 2 \alpha, \beta)$ & 1,1 & K \\
\hline$\left(21^{2}\right)$ & 5 & $x y, x z+y z, 0$ & $(\alpha, \alpha, \beta),(2 \alpha, \alpha+\beta, \gamma)$ & $1,1,1$ & $\neq$ \\
\hline$(31)$ & 6 & $x z, x^{2}-y z, 0$ & $(\alpha+\beta, 2 \alpha, 2 \beta),(\alpha+3 \beta, 2 \alpha+2 \beta, \gamma)$ & $1,1,1$ & $\Xi$ \\
\hline$(22)$ & 6 & $x^{2}, y z, 0$ & $(\alpha, \beta, \gamma),(2 \alpha, \beta+\gamma, \delta)$ & $1,1,2$ & $\neq$ \\
\hline (4) & 7 & $x z+y^{2}, x^{2}, 0$ & $(2 \alpha, \alpha+\beta, 2 \beta),(2 \alpha+2 \beta, 4 \alpha, \gamma)$ & $1,1,1$ & $\Xi$ \\
\hline$K$ & 8 & $y^{2}, z^{2}, 0$ & $(\beta, \alpha, \gamma),(2 \alpha, 2 \gamma, \delta)$ & $1,1,1,2$ & 0 \\
\hline$L$ & 8 & $x y, x z, 0$ & $(\alpha, \beta, \gamma),(\alpha+\beta, \alpha+\gamma, \delta)$ & $1,1,1,2$ & 0 \\
\hline$M$ & 9 & $y z, y^{2}, 0$ & $(\alpha, \beta, \gamma),(\beta+\gamma, 2 \beta, \delta)$ & $1,1,1,1$ & 0 \\
\hline \multicolumn{6}{|l|}{$\Sigma^{2}$} \\
\hline$S$ & 10 & $x y-z^{2}, 0,0$ & $(2 \alpha, 2 \beta, \alpha+\beta),(2 \alpha+2 \beta, \gamma, \delta)$ & $1,1,2,2$ & 0 \\
\hline$P L$ & 11 & $x y, 0,0$ & $(\alpha, \beta, \gamma),(\alpha+\beta, \delta, \epsilon)$ & $1,1,1,2,2$ & 0 \\
\hline$D L$ & 13 & $x^{2}, 0,0$ & $(\alpha, \beta, \gamma),(2 \alpha, \delta, \epsilon)$ & $1,1,1,2,2$ & 0 \\
\hline 0 & 18 & $0,0,0$ & $(\alpha, \beta, \gamma),(\delta, \epsilon, \kappa)$ & $1,1,2,2,3,3$ & 0 \\
\hline
\end{tabular}

The list of codimension> 1 orbits is given in the first 3 columns of Table 1 with the following conventions. Column 1 is the name of the orbit, column 2 is its codimension, and column 3 names three plane conics that span the image of $\phi \in \operatorname{Hom}\left(V^{*}, S^{2} U^{*}\right)$. Here we used the letters $x, y, z$ for the coordinates on $U$.

3.2. Equivariant classes. $G$-invariant subvarieties (e.g., orbit closures) represent cohomology classes in the equivariant cohomology ring of a $G$-representation. We want to determine the equivariant classes $[\bar{\eta}] \in H_{\mathrm{GL}(U) \times \mathrm{GL}(V)}^{*}(\mathbf{N o c}) \cong \mathbb{Z}\left[u_{1}, u_{2}, u_{3}, v_{1}, v_{2}, v_{3}\right]$ for the orbits $\eta \subset$ Noc. Here $u_{1}, u_{2}, u_{3}$ and $v_{1}, v_{2}, v_{3}$ denote the Chern classes of the groups $\mathrm{GL}(U)$ and $\mathrm{GL}(V)$ respectively. These classes contain a lot of geometric information as we will show later.

For the codimension $>1$ orbits we use the method of restriction equations of [Rim01, Thm.2.4], see also [FR04, Sect.3], so we need the symmetries of these orbits. More precisely, we need only a maximal torus of their stabilizer subgroups. These elementary calculations can be reduced to the level of Lie algebras. The results are summarized in the "symmetry" column of Table 1.

To explain the notation, consider the orbit $C$ represented by the net of conics

$$
\left(y^{2}+2 x z, 2 y z,-x^{2}\right) .
$$


The pair of matrices

$$
\left(\left(\begin{array}{ccc}
a^{2} & 0 & 0 \\
0 & a b & 0 \\
0 & 0 & b^{2}
\end{array}\right),\left(\begin{array}{ccc}
a^{2} b^{2} & 0 & 0 \\
0 & a b^{3} & 0 \\
0 & 0 & a^{4}
\end{array}\right)\right) \in \mathrm{GL}(U) \times \mathrm{GL}(V), \quad a, b \in \mathrm{GL}_{1}
$$

stabilize this net of conic. Since the codimension of $C$ is 2, the dimension of the stabilizer subgroup has to be 2 as well $(\operatorname{dim} G=\operatorname{dim} V=18)$, so we determined the maximal torus. This is the data that is encoded as $(2 \alpha, \alpha+\beta, 2 \beta),(2 \alpha+2 \beta, \alpha+3 \beta, 4 \alpha)$ in Table 1.

Theorem 3.1. Consider the $\mathrm{GL}(U) \times \mathrm{GL}(V)$ representation Noc. The Theorem of Restriction Equations [FR04, Thm. 3.5] determines all the $\mathrm{GL}(U) \times \mathrm{GL}(V)$ equivariant classes of the codimension $>1$ orbit closures, e.g., we have

- $[\bar{C}]=8\left(v_{1}-2 u_{1}\right)^{2}$,

- $[\bar{D}]=-3 u_{2}+3 v_{2}-16 u_{1} v_{1}+3 v_{1}^{2}+17 u_{1}^{2}$,

- $\left[\overline{D^{*}}\right]=12 u_{2}-3 v_{2}-20 u_{1} v_{1}+6 v_{1}^{2}+16 u_{1}^{2}$,

- $[\bar{E}]=3 u_{3}+3 v_{3}-3 u_{1} u_{2}+u_{2} v_{1}-6 u_{1} v_{1}^{2}+13 u_{1}^{2} v_{1}-2 u_{1} v_{2}-8 u_{1}^{3}+v_{1}^{3}$

- $\left[\overline{E^{*}}\right]=-24 u_{3}+3 v_{3}-24 u_{1} u_{2}+16 u_{2} v_{1}-16 u_{1} v_{1}^{2}+20 u_{1}^{2} v_{1}-6 v_{1} v_{2}+10 u_{1} v_{2}-8 u_{1}^{3}+4 v_{1}^{3}$

- $[\bar{F}]=2\left(v_{1}-2 u_{1}\right)\left(6 u_{1}^{2}-4 u_{1} v_{1}-6 u_{2}+3 v_{2}\right)$,

- $\left[\overline{F^{*}}\right]=2\left(v_{1}-2 u_{1}\right)\left(5 u_{1}^{2}-8 u_{1} v_{1}+9 u_{2}-3 v_{2}+3 v_{1}^{2}\right)$.

Proof. The proof does not follow from any general principle we are aware of, it is just an experimental fact. (The condition under which the Restriction Equations determine all equivariant classes in [FR04, Thm. 3.5] is that there are finitely many orbits, and each of them satisfy an Euler-class condition. For our representation there is a moduli of orbits.) The symmetry data of the table put constraints on the classes $[\bar{\eta}]$. One can write down all these constraints for each codimension $>1$ orbit $\eta$. A computer program shows that for each codimension $>1$ orbit there is only one equivariant class in $H^{*}(B(\mathrm{GL}(U) \times \mathrm{GL}(V)))$ satisfying the constraints.

For the family of codimension 1 orbits we look at the Wall-DuPlessis classification [WdP95] from an equivariant point of view.

The affine plane $N_{C}=\left\{\nu_{c, g}: c, g \in \mathbb{C}\right\}$, where

$$
\nu_{c, g}=\left(y^{2}+2 x z, 2 y z,-x^{2}+2 g\left(x z-y^{2}\right)+c z^{2}\right),
$$

is normal to the orbit $C$ at the point $\left(y^{2}+2 x z, 2 y z,-x^{2}\right)$. This plane is invariant under the action of the complex 2 -torus $T_{C}$ of (1). The $T_{C}$ action on $N_{C}$ has weights $2 \alpha-2 \beta$ and $4 \alpha-4 \beta$, corresponding to the weight vectors $\left(0,0, x z-y^{2}\right)$ and $\left(0,0, z^{2}\right)$. Hence, the orbits of $T_{C}$ on $N_{C}$ correspond to the parabolas with $\mu=\left(c: g^{2}\right) \in \mathbf{P}^{1}$ constant.

According to [WdP95] these parabolas are exactly the intersections of the codimension 1 Nocorbits with the normal slice $N_{C}$. We will refer to the orbit of $\nu_{c, g}$ with $\mu=\left(c: g^{2}\right)$ as $A_{\mu}$. In [Wal77] $A_{-9}$ is called $B$ and $A_{0}$ is called $B^{*}$. We will refer to a Noc-orbit representative lying in $N_{C}$ as a $c$-g-form. Recall the following Incidence Theorem.

Theorem 3.2. [FP09] Consider a Lie group $G$ acting on a vector space $V$ complex linearly. For $v \in V$ let $G_{v}$ denote the stabilizer subgroup of $v$. Let $S$ be a subgroup of $G_{v}$ and $N_{v}$ an $S$-invariant normal slice to the orbit $G v$ at $v$. Suppose that $\eta \subset V$ is a $G$-invariant subvariety. Then

$$
[\eta \subset V]_{S}=\left[\left(\eta \cap N_{v}\right) \subset N_{v}\right]_{S} .
$$

Theorem 3.3. The equivariant classes of the $A_{\mu}$ orbits are $4\left(v_{1}-2 u_{1}\right)$ for $\mu \neq \infty$ and $2\left(v_{1}-2 u_{1}\right)$ for $\mu=\infty$. 
Proof. If $\mu \neq \infty$, then the class $\left[\left(\mu g^{2}=c\right) \subset N_{C}\right]$ is equal to the weight of the $g=0$ direction. Hence we have $\left[\left(\mu g^{2}=c\right) \subset N_{C}\right]=4 \alpha-4 \beta$. For the curve $g=0$ we have

$$
\left[(g=0) \subset N_{C}\right]=2 \alpha-2 \beta .
$$

For the restriction homomorphism $r: H_{\mathrm{GL}(U) \times \mathrm{GL}(V)}^{*} \rightarrow H_{T_{C}}^{*}=\mathbb{Z}[\alpha, \beta]$, we have

$$
r\left(u_{1}\right)=2 \alpha+(\alpha+\beta)+2 \beta=3 \alpha+3 \beta
$$

and

$$
r\left(v_{1}\right)=(2 \alpha+2 \beta)+(\alpha+3 \beta)+4 \alpha=7 \alpha+5 \beta
$$

by (1). Hence, if $\left[A_{\mu}\right]=A u_{1}+B v_{1}(\mu \neq \infty)$, then according to Theorem 3.2, we have

$$
A(3 \alpha+3 \beta)+B(7 \alpha+5 \beta)=4 \alpha-4 \beta .
$$

The only solution is $A=-8, B=4$. For $\mu=\infty$ the calculation is similar.

Remark 3.4. From the equivariant classes of (cone) varieties one can calculate their degrees, see, e.g., [FNR05, Sec. 6] and Section 7. Therefore, Theorem 3.3 implies that the degree of the hypersurfaces given by the closures of the $A_{\mu}$ orbits is $4(1+1+1-2(0+0+0))=12$ in general, and 6 for $\mu=\infty$. This implies that the ring of invariants $R(\mathbf{N o c})$ on Noc is generated by a degree 6 and a degree 12 polynomial. We will give a full description of $R(\mathbf{N o c})$ in Section 5 .

\section{Thom POlynomials of CONTACT SINGUlaRities CORRESPONDING TO NETS OF CONICS}

Consider a polynomial map $g:\left(\mathbb{C}^{n}, 0\right) \rightarrow\left(\mathbb{C}^{p}, 0\right)$. In global singularity theory one studies its Thom polynomial $\mathrm{Tp}_{g} \in \mathbb{Z}\left[\alpha_{1}, \ldots, \alpha_{n}, \beta_{1}, \ldots, \beta_{p}\right]^{S_{n} \times S_{p}}$. Here $S_{n}$ permutes the $\alpha_{i}$ variables (the so-called "source Chern roots"), and $S_{p}$ permutes the $\beta_{j}$ variables (the so-called "target Chern roots").

To recall the definition of $\mathrm{Tp}_{g}$ we need to define contact equivalence. Consider the maximal ideal $\mathfrak{m}$ of the ring of formal power series $\mathbb{C}\left[\left[x_{1}, \ldots, x_{n}\right]\right]$. To any polynomial map $g:\left(\mathbb{C}^{n}, 0\right) \rightarrow$ $\left(\mathbb{C}^{p}, 0\right)$ (or, equivalently a jet in $\left.J^{k}(n, p)\right)$ we assign the ideal $I_{g}=\left(g_{1}, \ldots, g_{p}\right) \triangleleft \mathfrak{m}$ generated by the coordinate functions of $g$. We say that two jets are contact equivalent if their ideals are equivalent under a local holomorphic reparametrization of $\mathbb{C}^{n}$. The Thom polynomial $\mathrm{Tp}_{g}$ of the map $g$ is the $T=U(1)^{n} \times U(1)^{p}$-equivariant cohomology class represented by the set of jets contact equivalent to $g$, in the jet space $J^{k}(n, p)$ (where $k$ is high enough). Sets of contact equivalent jets will be called contact classes. They can either be defined by $g$ or by the ideal $I_{g}$.

We will assign two contact classes to a net of conics $f \in \operatorname{Hom}\left(S^{2} U, V\right)$. Let $f$ be represented by three degree- 2 polynomials $f_{1}, f_{2}, f_{3}$ in $x, y, z$ (see examples in Table 1 ). Consider the ideals $I_{f}=\left(f_{1}, f_{2}, f_{3}\right)$ and $I_{\tilde{f}}=\left(f_{1}, f_{2}, f_{3}\right)+(x, y, z)^{3}$, they define contact classes, $I_{f}$ for $p \geq 3$ and $I_{\tilde{f}}$ only for higher values of $p$.

We are mainly interested in the Thom polynomial defined by $I_{f}$ for $p=3$, since among these are the the smallest (lowest degree) Thom polynomials that can not be computed by previous methods ([Rim01]). However we need to make a detour to calculate Thom polynomials corresponding to $I_{\tilde{f}}$ first. The reason is explained in Section 4.1.

Theorem 4.1. Let $f \in \operatorname{Hom}\left(S^{2} U, V\right)$ be a $\Sigma^{0}$ net of conics, and let $[f] \in \mathbb{Z}\left[u_{1}, u_{2}, u_{3}, v_{1}, v_{2}, v_{3}\right]$ be the equivariant class of its $\mathrm{GL}(U) \times \mathrm{GL}(V)$-orbit considered in Section 3. Let $\sigma_{f}:\left(\mathbb{C}^{3}, 0\right) \rightarrow$ $\left(\mathbb{C}^{p}, 0\right)$ be a polynomial map with $I_{\tilde{f}}=I_{\sigma_{f}}$. Let $W=\left\{2 \alpha_{1}, 2 \alpha_{2}, 2 \alpha_{3}, \alpha_{1}+\alpha_{2}, \alpha_{1}+\alpha_{3}, \alpha_{2}+\alpha_{3}\right\}$. Then

$$
\operatorname{Tp}_{\sigma_{f}}=\prod_{i=1}^{3} \prod_{j=1}^{p}\left(\beta_{j}-\alpha_{i}\right) \cdot \sum_{H \subset W,|H|=3} \frac{\left.D_{H} \cdot[f]\right|_{H}}{e_{H}}
$$


where

$D_{H}=\prod_{w \notin H} \prod_{j=1}^{p}\left(\beta_{j}-w\right), \quad e_{H}=\prod_{w_{1} \notin H} \prod_{w_{2} \in H}\left(w_{2}-w_{1}\right),\left.\quad[f]\right|_{H}=\left.[f]\right|_{v_{i}=\sigma_{i}(H), u_{i}=\sigma_{i}\left(\alpha_{1}, \alpha_{2}, \alpha_{3}\right)}$.

In the last substitution $\sigma_{i}$ means the $i$ 'th elementary symmetric polynomial.

In Section 4.1 we will show how this Theorem follows from the "Localization Formula" [FR12, Thm 6.1].

Remark 4.2. Similar, and in some sense simpler formulas can be given for the non- $\Sigma^{0}$ nets. The corresponding polynomial maps belong to very large codimensional singularities, consequently their Thom polynomials are of very large degree, so we omit the description.

Theorem 4.1 gives the Thom polynomial in the Chern root variables $\alpha_{i}$ and $\beta_{j}$. A more compact way to write down these polynomials is in quotient variables, using the basis of Schur polynomials. To indicate that we use quotient variables we use the notation $\mathrm{tp}_{\sigma_{f}}$. The Schur polynomials $\Delta$ are defined by $\Delta_{\left(\lambda_{1}, \ldots, \lambda_{r}\right)}=\operatorname{det}\left(c_{\lambda_{i}+j-i}\right)_{r \times r}$; for example,

$$
\Delta_{(31)}=\operatorname{det}\left(\begin{array}{ll}
c_{3} & c_{4} \\
c_{0} & c_{1}
\end{array}\right)=c_{3} c_{1}-c_{4}
$$

and $c_{i}$ denotes the degree $i$ homogeneous part of $\frac{\prod_{j=1}^{p}\left(1+\beta_{j}\right)}{\prod_{i=1}^{3}\left(1+\alpha_{i}\right)}$. Note that this convention for Schur polynomials is that of, e.g., [FP98], which slightly differs from that of, e.g., [Mac98].

Theorem 4.1, and some calculation, gives that for example for $\sigma_{f}: \mathbb{C}^{3} \rightarrow \mathbb{C}^{4}$,

$$
\sigma_{f}(x, y, z)=\left(y^{2}+2 x z, 2 y z,-x^{2}+2 g\left(x z-y^{2}\right)+c z^{2}, z^{3}\right)
$$

we have

$$
\begin{array}{r}
\text { (3) } \operatorname{tp}_{\sigma_{f}}=8 \Delta_{(544111)}+4 \Delta_{(444211)}+16 \Delta_{(844)}+20 \Delta_{(6442)}+32 \Delta_{(64411)}+120 \Delta_{(6541)}+160 \Delta_{(655)}+ \\
16 \Delta_{(54421)}+32 \Delta_{(55411)}+40 \Delta_{(5542)}+80 \Delta_{(5551)}+80 \Delta_{(664)}+40 \Delta_{(7441)}+112 \Delta_{(754)},
\end{array}
$$

if $g \neq 0$ and half of it for $g=0$.

4.1. Fibered localization. Now we show how the arguments in [FR12] can be applied to prove Theorem 4.1, some familiarity of Sections 5, 6 of [FR12] are assumed. The localization formula of [FR12, Thm 6.1] is based on a generalization of the Atiyah-Bott-Berline-Vergne localization: the fibered localization formula of Vergne-Rossmann-Bérczi-Szenes ([BS12]). This formula calculates, from some fixed point data, the torus equivariant cohomology of a variety which is the birational image of a vector bundle $E \rightarrow Y$ such that the base $Y$ is a subvariety of a smooth manifold (everything is equipped with compatible torus actions).

For the proof of Theorem 4.1 we consider the correspondence variety

$$
C(Y)=\left\{(I, g) \in \operatorname{Gr}^{m}\left(J^{k}(n)\right) \times J^{k}(n, p) \mid I \in Y, I_{g} \subset I\right\},
$$

where $g \in J^{k}(n, p)$ is a jet, $m$ is the codimension of $I_{g} \triangleleft J^{k}(n), \operatorname{Gr}^{m}\left(J^{k}(n)\right)$ denotes the $m$ codimensional subspaces of the jet space $J^{k}(n)$ and $Y$ is the closure of the set of ideals equivalent to $I_{g}$. The first projection $C(Y) \rightarrow Y$ is a vector bundle, and it can be shown that the second projection is a birational map to the closure of the contact class of $g$. The variety $Y$ is contained in the manifold $\operatorname{Gr}_{3}\left(S^{2} \mathbb{C}^{3}\right)$ (for $f \in \Sigma^{i}$ it would be $\operatorname{Gr}_{3-i}\left(S^{2} \mathbb{C}^{3}\right)$ ). Hence, the fibered localization formula ([FR12, Prop 5.1 or Thm 6.1]) can be applied.

This formula expresses $\mathrm{Tp}_{\sigma_{f}}$ as a sum whose terms correspond to torus fixed points of $\operatorname{Gr}_{3}\left(S^{2} \mathbb{C}^{3}\right)$, that is, to the set $\{H \subset W,|H|=3\}$. The term corresponding to $H$ has a "fiber" 
component and a "horizontal" component. The fiber component is explicitly $D_{H}$, and the "horizontal" component, expressing the class of $Y$ at the fixed point $H$ is

$$
\left.[Y]\right|_{H}=\left.[f]\right|_{v_{i}=\sigma_{i}(H), u_{i}=\sigma_{i}\left(\alpha_{1}, \alpha_{2}, \alpha_{3}\right)} .
$$

Remark 4.3. What made this calculation very simple is the fact that $Y$ is contained in the Grassmannian $\operatorname{Gr}_{3}\left(S^{2} \mathbb{C}^{3}\right)$. For the ideals $I_{f}$ we are not aware of any similar simplification. However for $p=3$ we will calculate the Thom polynomials for some nets of conics in the next section.

4.2. Thom polynomials of equidimensional maps. Notice that for any $\sigma_{f}$ satisfying the condition of Theorem 4.1, $p$ is at least 4. It is possible to substitute however $p=3$ formally into the formula, and in some cases we can interpret the result as a Thom polynomial. This phenomenon was called the "small $p$ case" in [FR12, §12]. According to the previous section we need to check that the second projection of the correspondence variety (4) for $Y$ being the closure of the set of ideals equivalent to $I_{\tilde{f}}$ is a birational map to the closure of the contact class of $f \in J^{2}(3,3)$. Obviously $f$, therefore its contact class, is in the image, so the following two conditions will imply birationality:

(1) The second projection is generically one-to-one.

(2) The dimension of the contact class of $f$ equals to that of the correspondence variety $C(Y)$.

Condition (1) is equivalent to the condition that there is only one ideal equivalent to $I_{\tilde{f}}$ containing $I_{f}$ : the ideal $I_{\tilde{f}}$ itself. This is satisfied for all $f$, since any ideal equivalent to $I_{\tilde{f}}$ should contain the invariant ideal $(x, y, z)^{3}$. By calculating the dimension of the correspondence variety we can see that the second condition is equivalent to the condition that the codimension of the contact class of $f$ in $J^{2}(3,3)$ is $\operatorname{deg}[f]+9$.

We give 3 examples when the second condition is also satisfied (see [WdP95, p. 315]).

1. The Thom polynomial of the germ $h_{c, g}: \mathbb{C}^{3} \rightarrow \mathbb{C}^{3}$,

$$
h_{c, g}(x, y, z)=\left(y^{2}+2 x z, 2 y z,-x^{2}+2 g\left(x z-y^{2}\right)+c z^{2}\right)
$$

is

$$
\operatorname{tp}_{h_{c, g}}=8 \Delta_{(433)}+4 \Delta_{(3331)}
$$

if $g \neq 0$ and half of it for $g=0$. The singularities $h_{c, g}$ form the smallest codimensional example of a family of non-equivalent contact singularities $\left(\mathbb{C}^{n}, 0\right) \rightarrow\left(\mathbb{C}^{n}, 0\right)$ ([Mat71]). Because of the presence of this continuous modulus in the classification of singularities, the method of [Rim01] calculating Thom polynomials broke down at codimension 9. (In [FR12] a different representation of $h_{c, g}$ is used, namely $\left(x^{2}-\lambda y z, y^{2}-\lambda x z, z^{2}-\lambda x y\right)$. This representation is less adapted to our purposes, in general 12 different values of $\lambda$ correspond to the same orbit, and the singularities corresponding to $B$ and $B^{*}$ cannot be written in this form.)

2 and 3: The singularities corresponding to nets from the orbits $D$ and $E$ are denoted by $K D$ and $K E$ in [WdP95, p. 315]. Hence [FR12, Section 7] implies:

Theorem 4.4. The Thom polynomials of maps $\left(\mathbb{C}^{n}, 0\right) \rightarrow\left(\mathbb{C}^{n}, 0\right)$ of type $K D$ and $K E$ are

$$
\begin{aligned}
\operatorname{tp}_{K D}= & 3 \Delta_{(33311)}+6 \Delta_{(3332)}+14 \Delta_{(443)}+16 \Delta_{(4331)}+17 \Delta_{(533)}, \\
\operatorname{tp}_{K E}= & \Delta_{(333111)}+2 \Delta_{(33321)}+4 \Delta_{(3333)}+6 \Delta_{(43311)}+8 \Delta_{(4332)}+ \\
& +14 \Delta_{(4431)}+8 \Delta_{(444)}+13 \Delta_{(5331)}+19 \Delta_{(543)}+8 \Delta_{(633)} .
\end{aligned}
$$


M. DOMOKOS, L. M. FEHÉR, AND R. RIMÁNYI

Remark 4.5. For some pair of contact singularities only condition (2) is satisfied. Examples are given in [FR12, §12]. In such cases the localization formula gives the Thom polynomial multiplied by the number of sheets of the second projection. (The simplest example we are aware of is the singularities $\tilde{f}=\left(x^{3}, x y, y^{2}\right)$ and $f=(x y)$. In this case we have two sheets: the ideal $\left(x^{2}, x y, y^{3}\right)$ also contains $(x y)$.)

Remark 4.6. A polynomial map is called equidimensional, if the dimension of its source is the same as the dimension of its target. Thom polynomials of equidimensional polynomial maps were calculated in [Rim01] up to degree 8. The results above on the Thom polynomials of $h_{c, g}, K D$, and $K E$ are essentially the first examples beyond degree 8 . Notice that we calculated the Thom polynomials of these singularities only for $p=3$, as opposed to the singularities corresponding to $I_{\tilde{f}}$ where we obtained the Thom polynomial for all $p$.

4.3. Duality and the Kazarian theory. Recently M. Kazarian developed a method ([Kaz]) to calculate Thom polynomials, in particular Section 8.8 of [Kaz] deals with nets of conics. His approach is dual to the method of [FR12] in the sense that it uses the quotient algebra as opposed to the ideal of the singularity. For a singularity corresponding to a $\Sigma^{0}$ net $f$ in the above sense the quotient algebra is $Q_{f}=J^{2}(U, V) /\left(f_{1}, f_{2}, f_{3}\right)$. This is a graded algebra of graded degree $(3,3)$, so the multiplication is determined by a linear map $m \in \operatorname{Hom}\left(S^{2} U^{*}, \operatorname{ker} f\right)$. The map $m$ is the apolar of $f$ in the terminology of [Wal77]. The input of the Kazarian method is the equivariant class of $m$. The apolar of a net lives in the dual representation, however, by fixing a basis of $U$ we can identify these spaces. The identification depends on the basis but a basis change does not change the orbit, so we get a well defined duality on the $\Sigma^{0}$ orbits. Wall denotes this duality by *. It is also true (and easy to see) that the equivariant classes of the orbits are the same up to sign in the dual representation, however in general the dual orbit has different equivariant class, see, e.g., $[D] \neq\left[D^{*}\right]$ and consequently the Thom polynomials of the corresponding singularities are also different. Using the Kazarian theory we were able to double check our results.

\section{INVARIANT THEORY OF NETS OF CONICS}

Most of the results in this section are known (see [Wal77], [Wal10], [WdP95] [Vin76] and [AN02]), but we also would like to show how equivariant theory leads to these results with the hope that it can be applied in a more general context.

5.1. The ring of (semi) invariants. Suppose that the Lie group $G$ acts on the vector space $W$ and $\hat{G}$ is the character group of $G$. We say that $f \in \mathbb{C}[W]$ is a relative invariant corresponding to the character $\chi \in \hat{G}$ (i.e., $f \in R_{\chi}(W)$ ) if for all $v \in W$ and $g \in G$

$$
f(g v)=\chi(g) f(v) .
$$

The ring of semi-invariants is $R(W):=\bigoplus_{\chi \in \hat{G}} R_{\chi}(W)$.

Note that an element of $R(W)$ is not necessarily a relative invariant for any $\chi \in \hat{G}$. Semiinvariants of $G$ are always invariants of the commutator subgroup $G^{\prime}$, but in general the ring of invariants of $G^{\prime}$ can be bigger. In the following two examples, however, they coincide.

(a) The $\mathrm{GL}(U) \times \mathrm{GL}(V)$-action on Noc: Any character of $\mathrm{GL}(U) \times \mathrm{GL}(V)$ is of the form $\chi_{a, b}(g, h):=\operatorname{det}^{a}(g) \operatorname{det}^{b}(h)$. If $f \in R(\mathbf{N o c})$ is homogeneous of degree $l=3 d$, then

$$
f((\lambda I, \mu I) v)=f\left(\lambda^{-2} \mu v\right)=\lambda^{-2 l} \mu^{l} f(v)=\operatorname{det}^{-2 d}(\lambda I) \operatorname{det}^{d}(\mu I) f(v),
$$


therefore $f$ is a relative invariant corresponding to the character $\chi_{-2 d, d}$. (In GIT language we see that there is a unique linearization for GIT-quotient.) In other words

$$
R(\mathbf{N o c})=\bigoplus_{d \in \mathbb{N}} R_{\chi_{-2 d, d}}(\mathbf{N o c})
$$

In this case all characters are determinants, so $R(\mathbf{N o c})$ coincides with the ring of absolute invariant polynomials for the $\mathrm{SL}_{3} \times \mathrm{SL}_{3}$-action on Noc.

(b) As we discussed before, the maximal torus of the stabilizer of the net $\left(y^{2}+2 x z, 2 y z,-x^{2}\right)$ is $\mathrm{GL}_{1} \times \mathrm{GL}_{1}$. It acts on the normal space $N_{C}$ to its orbit with weights $4 \alpha-4 \beta$ and $2 \alpha-2 \beta$. With respect to this action we have

$$
R\left(N_{C}\right)=\bigoplus_{d \in \mathbb{N}} R_{\chi_{2 d,-2 d}}\left(N_{C}\right)=\mathbb{C}\left[N_{C}\right]=\mathbb{C}[c, g],
$$

where $c \in R_{\chi_{4,-4}}\left(N_{C}\right)$ and $g \in R_{\chi_{2,-2}}\left(N_{C}\right)$. Consider the restriction map $i^{*}: R(\mathbf{N o c}) \rightarrow$ $R\left(N_{C}\right)$. From the first line of Table 1 (equivalently, from (1)) one sees that $i^{*}$ maps $R_{\chi_{-2 d, d}}\left(\right.$ Noc) into $R_{\chi_{d,-d}}\left(N_{C}\right)$.

We claim that $i^{*}: R(\mathbf{N o c}) \rightarrow R\left(N_{C}\right)$ is injective. Indeed, according to the splitting above it is enough to show that it is injective on $R_{\chi_{-2 d, d}}(\mathbf{N o c})$ for any given $d$, which follows from the fact that the values of a relative invariant $f$ on $N_{C}$ determine its values on $(\mathrm{GL}(U) \times \mathrm{GL}(V)) \cdot N_{C}$ via (5) and $(\mathrm{GL}(U) \times \mathrm{GL}(V)) \cdot N_{C}$ is dense in Noc. Now we prove that the homomorphism $i^{*}$ is also surjective, by finding relative invariants of Noc mapped to $c$ and $g$.

5.2. The determinant map. Composing nets with the determinant $S^{2} U^{*} \rightarrow \mathbb{C}$ (well defined up to a scalar factor) we get a degree 3 polynomial map $\delta: \operatorname{Noc} \rightarrow S^{3} V$. This map is GL $(U) \times$ $\mathrm{GL}(V)$-equivariant if we let $\mathrm{GL}(U)$ act on $S^{3} V$ as scalars by the second tensor power of the determinant representation. Consequently we have a homomorphism $\delta^{*}: R\left(S^{3} V\right) \rightarrow R($ Noc $)$. Let us review now the invariant theory of the plane cubics $S^{3} \mathbb{C}^{3}$ which was one of the first achievements of the early invariant theory.

Theorem 5.1. [Aro50] There are invariants a, $b$ of $S^{3} \mathbb{C}^{3}$ of degree 4 and 6 , respectively such that $R\left(S^{3} \mathbb{C}^{3}\right) \cong \mathbb{C}[a, b]$ and every smooth plane cubic $\gamma$ can be transformed (using the $\mathrm{GL}_{3}$-action) into the Weierstrass-form:

$$
y^{2} z+x^{3}+a(\gamma) x z^{2}+b(\gamma) z^{3} .
$$

Remark 5.2. The Weierstrass-form is analogous to the $c$-g-form of nets of conics from Section 3 . The subset $\left\{y^{2} z+x^{3}+a x z^{2}+b z^{3}: a, b \in \mathbb{C}\right\}$ is a normal slice to the orbit of the cuspidal cubic $y^{2} z+x^{3}$. This observation was used in [Köm03] to calculate the equivariant classes for plane cubics. The choice of these orbits is not accidental. Their closure is the nullcone, so the normal slice intersects all invariant hypersurfaces.

Consider now the determinant of the $c$ - $g$-form $\nu_{c, g}=\left(y^{2}+2 x z, 2 y z,-x^{2}+2 g\left(x z-y^{2}\right)+c z^{2}\right)$. Considering the three $3 \times 3$ matrices of the three components of $\nu_{c, g}$ we obtain

$$
\begin{aligned}
\delta\left(\nu_{c, g}\right) & =\operatorname{det}\left((-x)\left(\begin{array}{ccc}
0 & 0 & 1 \\
0 & 1 & 0 \\
1 & 0 & 0
\end{array}\right)+(-y)\left(\begin{array}{lll}
0 & 0 & 0 \\
0 & 0 & 1 \\
0 & 1 & 0
\end{array}\right)+z\left(\begin{array}{ccc}
-1 & 0 & g \\
0 & -2 g & 0 \\
g & 0 & c
\end{array}\right)\right) \\
& =y^{2} z+x^{3}+\left(c-3 g^{2}\right) x z^{2}+2 g\left(c+g^{2}\right) z^{3} .
\end{aligned}
$$

Here we parameterized $\mathbb{C}^{3}$ with $-x,-y, z$ to obtain our result, the Weierstrass form, without sign changes. Therefore $i^{*} \delta^{*} a=c-3 g^{2}$ and $i^{*} \delta^{*} b=2 g\left(c+g^{2}\right)$. We denote the degree 12 invariant $-48 \delta^{*} a$ by $J_{12}$. 


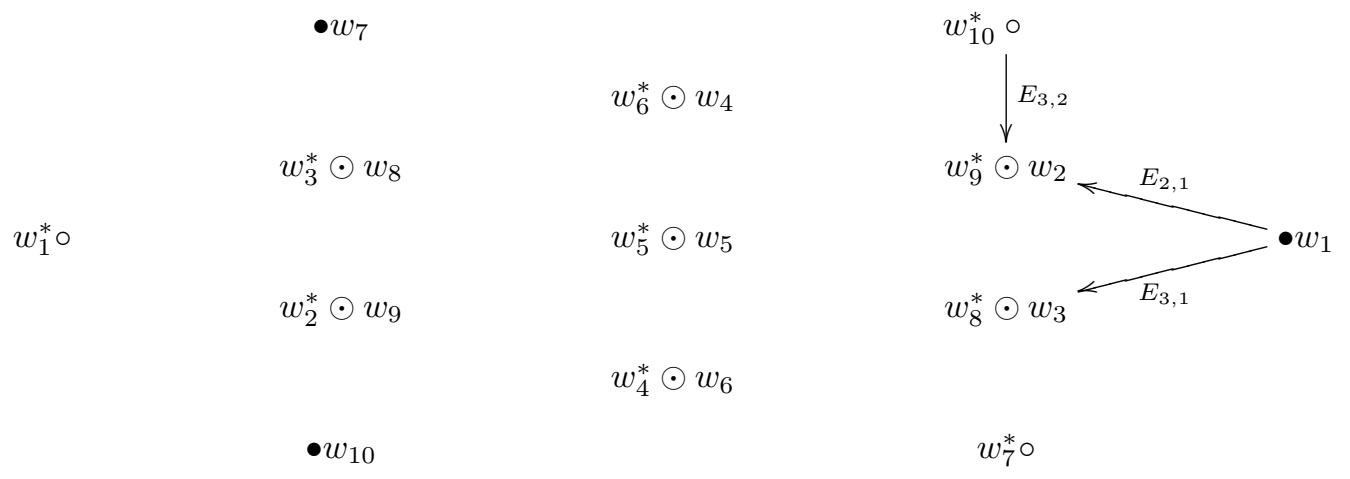

Figure 1. The 20 weights of $\mathbf{P l}$

To complete the calculation of $R$ (Noc) we need to find the degree 6 invariant which restricts to $g$ on the normal slice $N_{C}$. This is a straightforward job with a computer, but using a geometric idea it can be done by hand.

5.3. The Plücker map and the invariant $I_{2}$. We have a degree $3 \mathrm{GL}(U) \times \mathrm{GL}(V)$-equivariant map

$$
\psi: \mathbf{N o c}=\operatorname{Hom}\left(S^{2} U, V\right) \stackrel{\bigwedge^{3}}{\rightarrow} \operatorname{Hom}\left(\bigwedge^{3} S^{2} U, \bigwedge^{3} V\right) \cong \bigwedge^{3} S^{2} U^{*} \otimes \bigwedge^{3} V .
$$

We will call $\mathbf{P l}:=\bigwedge^{3} S^{2} U^{*} \otimes \bigwedge^{3} V$ the Plücker space.

Picking a basis for $V^{*}$, to each net we can associate a triple $M=\left(M_{1}, M_{2}, M_{3}\right)$ of conics, and $\psi$ sends $M$ to $M_{1} \wedge M_{2} \wedge M_{3} \in \wedge^{3} S^{2} U^{*}$. The image of $\psi$ is the cone of the Grassmannian $\operatorname{Gr}_{3}\left(S^{2} U^{*}\right)$. Our next goal is to show that the representation $\mathbf{P l}$ has a degree 2 invariant $I_{2}$ which pulls back to a degree 6 invariant $J_{6}$ of Noc.

Let $\xi, \eta, \nu$ be a basis in $U^{*}$ and $x, y, z$ the corresponding dual basis in $U$. Write $E_{i j}$ for the element of $\mathfrak{s l}(U)$ mapping the $i$ th basis vector of $U$ to the $j$ th basis vector and annihilating the other two basis vectors.

Consider the basis $e_{1}:=\xi^{2}, e_{2}:=\xi \eta, e_{3}:=\xi \nu, e_{4}:=\eta^{2}, e_{5}:=\eta \nu, e_{6}:=\nu^{2}$ in $S^{2} U^{*}$, and set $e_{i j k}:=e_{i} \wedge e_{2} \wedge e_{3} \in \wedge^{3} S^{2}\left(U^{*}\right)$. Under the natural identification of $S^{2} U$ with the dual of $S^{2}\left(U^{\star}\right)$, the basis in $S^{2}(U)$ dual to $e_{1}, \ldots, e_{6}$ is $t_{1}:=x^{2}, t_{2}:=2 x y, t_{3}:=2 x z, t_{4}:=y^{2}, t_{5}:=2 y z$, $t_{6}:=z^{2}$. Then $t_{i j k}:=t_{i} \wedge t_{j} \wedge t_{k} \in \bigwedge^{3} S^{2}(U)$ is the basis dual to $e_{i j k}$. In particular, $t_{i j k}(\pi(M))$ is the $3 \times 3$ minor corresponding to the $(i, j, k)$ columns of the $3 \times 6$ matrix of the net $M$ viewed as a linear map from $V^{*}$ to $S^{2} U^{*}$, with respect to the chosen bases.

As an $\mathrm{SL}(U)$-representation $\mathbf{P l} \cong S^{3}(U) \oplus S^{3}\left(U^{*}\right)$. This follows for example from the calculations below or by calculating the weights of Pl. (See Figure 1, picturing the weights of $\mathbf{P l}$, denoting the weights of $S^{3}(U)$ by dots and the weights of $S^{3}\left(U^{*}\right)$ by circles. We also indicated the weight vectors spanning the corresponding weight spaces.)

Denoting by $W$ the 10-dimensional $\mathrm{SL}(U)$-module $S^{3}(U)$ we have

$$
S^{2} \mathbf{P l}^{*} \cong S^{2} W \oplus S^{2} W^{*} \oplus W \otimes W^{*} .
$$

The first two summands do not contain the trivial SL $(U)$-module (say by the theorem of Aronhold on the invariants of ternary cubic forms), and the third summand contains one copy of the trivial representation by Schur's Lemma, spanned by $w_{1} w_{1}^{*}+\cdots+w_{10} w_{10}^{*}$, where $w_{1}, \ldots, w_{10}$ is a basis of $W$ and $w_{1}^{*}, \ldots, w_{10}^{*}$ is the corresponding dual basis in $W^{*}$. Table 2 contains explicit elements $w_{1}, \ldots, w_{10} \in \bigwedge^{3} S^{2}(U)$ spanning an $\mathfrak{s l}(U)$-summand isomorphic to $W$. Clearly $x^{2} \wedge x y \wedge x z$ 
is a highest weight vector generating an $\mathfrak{s t}(U)$-module isomorphic to $W$. The $w_{2}, \ldots, w_{10}$ are obtained by applying successively the operators $E_{21}, E_{31} \in \mathfrak{s t}(U)$, as indicated in Table 2 .

Recall that the action of $E_{i, j}$ is the sum of the replacements of each occurrence of the $j$ th basis vector to the $i$ th one, e.g.,

$E_{3,1}\left(x^{2} \wedge x y \wedge x z\right)=2 x z \wedge x y \wedge x z+x^{2} \wedge y z \wedge x z+x^{2} \wedge x y \wedge z^{2}=x^{2} \wedge x y \wedge z^{2}-x^{2} \wedge x z \wedge y z$.

Up to non-zero scalars $w_{10}^{*}$ in Table 2 is the only weight vector whose weight is opposite to the weight of the lowest weight element $w_{10}$ in $W$, therefore it must be a highest weight vector generating a submodule isomorphic to $W^{*}$ in $\bigwedge^{3} S^{2} U^{*}$. Applying successively appropriate elements of $\mathfrak{s l}(U)$ to $w_{10}^{*}$ one computes $w_{2}^{*}, \ldots, w_{10}^{*} \in \Lambda^{3} S^{2} U^{*}$. For example, by the left column of the table we have $w_{10}=E_{31} w_{6}$, hence $w_{6}^{*}=-E_{31} w_{10}^{*}$. In addition to the information in the left column of the table we need also the relations

$$
w_{8}=\frac{1}{3} E_{32} w_{7}, \quad w_{9}=\frac{1}{2} E_{32} w_{8}, \quad \text { and } \quad w_{10}=E_{32} w_{9} .
$$

With the $w_{i}, w_{i}^{*}$ given in Table 2 (for example, $w_{2}=-x^{2} \wedge x z \wedge y^{2}+x^{2} \wedge x y \wedge y z=-\frac{1}{2} t_{134}+\frac{1}{4} t_{125}$ ), we have the equality

$$
\begin{aligned}
-8 I_{2}:=8 \sum_{i=1}^{10} w_{i} w_{i}^{*} & =t_{235}^{2}-8 t_{146}^{2} \\
& -8 t_{134} t_{346}+8 t_{126} t_{246}+8 t_{145} t_{156} \\
& +6 t_{123} t_{456}-6 t_{136} t_{245}+6 t_{124} t_{356} \\
& -4 t_{125} t_{256}+4 t_{135} t_{345}-4 t_{234} t_{236} \\
& +2 t_{134} t_{256}-2 t_{125} t_{346}+2 t_{135} t_{246}-2 t_{126} t_{345} \\
& +2 t_{145} t_{236}+2 t_{156} t_{234}-2 t_{146} t_{235} .
\end{aligned}
$$

Remark 5.3. (i) Set $u_{i j k}:=t_{i j k} \circ \psi$, so $u_{i j k}$ is an element of the coordinate ring of Noc. Recall a classical result of Sylvester (see page 365 in [Sal79] or [Giz07]), asserting (after a change to our coordinate system) that

$$
\begin{aligned}
-8 \theta & :=u_{235}^{2}-8 u_{146}^{2} \\
& +4 u_{146} u_{235}+4 u_{135} u_{345}-4 u_{125} u_{256}-4 u_{234} u_{236} \\
& +8 u_{145} u_{156}-8 u_{134} u_{346}+8 u_{126} u_{246} \\
& +8 u_{123} u_{456}-8 u_{136} u_{245}+8 u_{124} u_{356}
\end{aligned}
$$

is an $\mathrm{SL}(U)$-invariant on Noc. We thank I. Dolgachev for bringing this reference to our attention. One can easily verify using the straightening algorithm (cf. Section 13.2.2 in [Pro07]) that $J_{6}:=I_{2} \circ \psi$ coincides with Salmon's $\theta$. Notice that one cannot reconstruct $I_{2}$ from $\theta$ since $\psi^{*}$ has a kernel, generated by the Plücker relations. To the best of our knowledge formula (6) for $I_{2}$ is new.

(ii) It is proved in [Vin76] that the ring of $\mathrm{SL}\left(\mathbb{C}^{3}\right) \times \mathrm{SL}\left(\mathbb{C}^{3}\right) \times \mathrm{SL}\left(\mathbb{C}^{3}\right)$-invariants in $\mathbb{C}^{3} \otimes \mathbb{C}^{3} \otimes \mathbb{C}^{3}$ is generated by three algebraically independent elements of degree 6,9 , and 12 . An alternative way to construct the invariant $J_{6}$ (not as a pullback from $\mathbf{P l}$ ) is to restrict the degree 6 generator to the subspace of $\mathbb{C}^{3} \otimes \mathbb{C}^{3} \otimes \mathbb{C}^{3}$ corresponding to symmetric matrix triples. Explicit formulae for the three generators can be found in [BLT04] or [DD12].

One can calculate that

$\psi\left(\nu_{c, g}\right)=\left(y^{2}+2 x z\right) \wedge 2 y z \wedge\left(x^{2}+2 g\left(x z-y^{2}\right)+c z^{2}\right)=12 g e_{345}+2 c\left(e_{456}+2 e_{356}\right)-2 e_{145}-4 e_{135}$, 
TABLE 2. Un-normalized generators

\begin{tabular}{|ll|l|}
\hline$w_{1}=x^{2} \wedge x y \wedge x z$ & $w_{10}^{*}$ & $=x^{2} \wedge x y \wedge y^{2}$ \\
\hline$w_{2}=E_{21} w_{1}=-x^{2} \wedge x z \wedge y^{2}+x^{2} \wedge x y \wedge y z$ & $w_{9}^{*}=-E_{32} w_{10}^{*}=-x^{2} \wedge x z \wedge y^{2}-2 x^{2} \wedge x y \wedge y z$ \\
\hline$w_{3}=E_{31} w_{1}=x^{2} \wedge y z \wedge x z+x^{2} \wedge x y \wedge z^{2}$ & $w_{8}^{*}=-\frac{1}{2} E_{32} w_{9}^{*}=2 x^{2} \wedge x z \wedge y z+x^{2} \wedge x y \wedge z^{2}$ \\
\hline$w_{4}=E_{21} w_{2}=2 x y \wedge y^{2} \wedge x z+2 x^{2} \wedge y^{2} \wedge y z$ & $w_{7}^{*}=-\frac{1}{3} E_{32} w_{8}^{*}=-x^{2} \wedge x z \wedge z^{2}$ \\
\hline$w_{5}=E_{31} w_{2}=x^{2} \wedge y^{2} \wedge z^{2}+2 x z \wedge x y \wedge y z$ & $w_{6}^{*}=-E_{31} w_{10}^{*}=2 x y \wedge x z \wedge y^{2}+x^{2} \wedge y^{2} \wedge y z$ \\
\hline$w_{6}=E_{31} w_{3}=2 x^{2} \wedge y z \wedge z^{2}+2 x z \wedge x y \wedge z^{2}$ & $w_{5}^{*}=-E_{31} w_{9}^{*}=-4 x y \wedge x z \wedge y z-x^{2} \wedge y^{2} \wedge z^{2}$ \\
\hline$w_{7}=E_{21} w_{4}=6 x y \wedge y^{2} \wedge y z$ & $w_{4}^{*}=-E_{21} w_{7}^{*}=2 x y \wedge x z \wedge z^{2}+x^{2} \wedge y z \wedge z^{2}$ \\
\hline$w_{8}=E_{31} w_{4}=2 x y \wedge y^{2} \wedge z^{2}+2 x z \wedge y^{2} \wedge y z$ & $w_{3}^{*}=-E_{31} w_{6}^{*}=2 x y \wedge y^{2} \wedge z^{2}-4 x z \wedge y^{2} \wedge y z$ \\
\hline$w_{9}=E_{31} w_{5}=2 x y \wedge y z \wedge z^{2}+2 x z \wedge y^{2} \wedge z^{2}$ & $w_{2}^{*}=-E_{21} w_{4}^{*}=2 x z \wedge y^{2} \wedge z^{2}-4 x y \wedge y z \wedge z^{2}$ \\
\hline$w_{10}=E_{31} w_{6}=6 x z \wedge y z \wedge z^{2}$ & $w_{1}^{*}=-E_{21} w_{2}^{*}=6 y^{2} \wedge y z \wedge z^{2}$ \\
\hline
\end{tabular}

and hence, for $J_{6}=I_{2} \circ \psi$ we have $J_{6}\left(\nu_{c, g}\right)=I_{2}\left(\psi\left(\nu_{c, g}\right)\right)=24 \mathrm{~g}$. This concludes our proof of the following known theorem (see [AN02], [Vin76], where the ring of invariants of Noc is identified with the ring of invariants of a finite complex pseudo-reflection group):

Theorem 5.4. The ring of invariants of Noc is freely generated by $J_{6}$ and $J_{12}$.

5.4. A geometric interpretation of the splitting of Pl. Following C.T.C. Wall [Wal10] we can interpret the projection maps from $\mathbf{P l}$ to its irreducible factors.

5.4.1. The Jacobi map. A net $\varphi$ is a linear map from $S^{2} U$ to $V$, alternatively a quadratic map from $U$ to $V$. Its derivative at $u \in U$ is a linear map $d_{u} \varphi: T_{u} U \rightarrow T_{u} V$. Since tangent spaces of a vector space can be canonically identified with the vector space itself and $d_{u} \varphi$ is linear in $u$, the derivative $d \varphi$ defines a linear map from $U$ to $\operatorname{Hom}(U, V)$. We also have the degree 3 determinant map

$$
\operatorname{det}: \operatorname{Hom}(U, V) \stackrel{\Lambda^{3}}{\rightarrow} \operatorname{Hom}\left(\bigwedge^{3} U, \bigwedge^{3} V\right) \cong \bigwedge^{3} U^{*} \otimes \bigwedge^{3} V
$$

which can be composed with $d \varphi$ to obtain a degree $3 \operatorname{map} \operatorname{Jac}(\varphi)$ from $U$ to $\bigwedge^{3} U^{*} \otimes \bigwedge^{3} V$. We can also consider Jac as a map

$$
\mathrm{Jac}: \text { Noc } \rightarrow S^{3} U^{*} \otimes \bigwedge^{3} U^{*} \otimes \bigwedge^{3} V .
$$

The map Jac factors through the Plücker map providing a linear projection

$$
\pi_{1}: \mathbf{P l} \rightarrow S^{3} U^{*} \otimes \bigwedge^{3} U^{*} \otimes \bigwedge^{3} V
$$

Picking a basis in $V^{*}$ we can identify Jac with the Jacobian covariant Jac : $\bigoplus^{3} S^{2}\left(U^{*}\right) \rightarrow S^{3}\left(U^{*}\right)$, which is a joint covariant of triples of conics defined as

$$
\operatorname{Jac}(M):=\operatorname{det}\left(\begin{array}{ccc}
\partial_{\xi} M_{1} & \partial_{\xi} M_{2} & \partial_{\xi} M_{3} \\
\partial_{\eta} M_{1} & \partial_{\eta} M_{2} & \partial_{\eta} M_{3} \\
\partial_{\nu} M_{1} & \partial_{\nu} M_{2} & \partial_{\nu} M_{3}
\end{array}\right)
$$

(recall that $\xi, \nu, \eta$ is our basis in $U^{*}$, and the $M_{i}$ are homogeneous quadratic polynomials in $\xi, \eta, \nu)$. Now Jac is an alternating trilinear function in $M_{1}, M_{2}, M_{3}$, hence it factors through an $\mathrm{SL}(U)$-equivariant linear map

$$
\pi_{1}: \mathbf{P l} \rightarrow S^{3}\left(U^{*}\right)
$$


It maps the basis vector $e_{i j k} \in \mathbf{P l}$ to the $3 \times 3$ minor corresponding to the $i, j, k$ columns of the matrix

$$
\left(\begin{array}{cccccc}
2 \xi & \eta & \nu & 0 & 0 & 0 \\
0 & \xi & 0 & 2 \eta & \nu & 0 \\
0 & 0 & \xi & 0 & \eta & 2 \nu
\end{array}\right)
$$

(the columns contain the partial derivatives for each of $\left.\xi^{2}, \xi \eta, \xi \nu, \nu^{2}, \eta \nu, \nu^{2}\right)$. Recall that $x^{3}, 3 x^{2} y$, $\ldots, 6 x y z, \ldots$ is the basis in $S^{3}(U)$ dual to the basis $\xi^{3}, \xi^{2} \eta, \ldots, \xi \eta \nu, \ldots$ of $S^{3}\left(U^{*}\right)$. Now $\pi_{1}^{*}$ embeds $S^{3}\left(U^{*}\right)^{*} \cong S^{3}(U)$ into $\mathbf{P l}^{*}$. For example, $\pi_{1}\left(e_{123}\right)=2 \xi^{3}$, and no other $\pi_{1}\left(e_{i j k}\right)$ contains the monomial $\xi^{3}$. This shows that $\pi_{1}^{*}\left(x^{3}\right)=2 t_{123}=8 w_{1}$ (where $w_{1}$ is the element given in Table 2). Similarly $\xi^{2} \eta$ is contained only in $\pi_{1}\left(e_{125}\right)=2 \xi^{2} \eta$ and $\pi_{1}\left(e_{134}\right)=-4 \xi^{2} \eta$. It follows that $\pi_{1}^{*}\left(3 x^{2} y\right)=2 t_{125}-4 t_{134}=8 w_{2}$. One checks in the same way that the basis $x^{3}, 3 x^{2} y, \ldots 6 x y z, \ldots, z^{3}$ of $S^{3}(U)$ is mapped under $\pi_{1}^{*}$ onto $8 w_{1}, \ldots, 8 w_{10}$ (cf. Table 2 ).

\subsubsection{The dual Jacobi map. We have a degree 2 map}

$$
\operatorname{Hom}\left(\mathbb{C}^{2}, U\right) \stackrel{S^{2}}{\rightarrow} \operatorname{Hom}\left(S^{2} \mathbb{C}^{2}, S^{2} U\right) .
$$

Composing with a net $\varphi$ and choosing an element in $\operatorname{Hom}\left(\mathbb{C}^{2}, U\right)$ we get a linear map in $\operatorname{Hom}\left(S^{2} \mathbb{C}^{2}, V\right)$. Taking its determinant we get a degree 6 map from $U \oplus U \cong \operatorname{Hom}\left(\mathbb{C}^{2}, U\right)$ to the one-dimensional vector space $L=\operatorname{Hom}\left(\bigwedge^{3} S^{2} \mathbb{C}^{2}, \bigwedge^{3} V\right) \cong \bigwedge^{3} V$. (As a representation of $\mathrm{GL}(U)$ the line $L$ is isomorphic to the trivial one-dimensional representation.) Notice that this map factors through $\bigwedge^{2} U$, providing a degree 3 map from $\bigwedge^{2} U$ to $L$. Varying $\varphi$ we end up with a degree 3 map from Noc $\rightarrow S^{3} \bigwedge^{2} U^{*} \otimes L$ which factors through the Plücker map $\psi$. Now notice that $\bigwedge^{2} U^{*} \cong U \otimes \bigwedge^{3} U^{*}$. Hence we defined a linear map

$$
\pi_{2}: \mathbf{P l} \rightarrow S^{3} U \otimes\left(\bigwedge^{3} U^{*}\right)^{3} \otimes \bigwedge^{3} V .
$$

Notice that the GL $(V)$-action played no active role in the projections $\pi_{1}$ and $\pi_{2}$ as it was expected from the abstract splitting of the representation $\bigwedge^{3} S^{2} U^{*}$.

As an SL $(U)$-equivariant linear map $\pi_{2}: \mathbf{P l} \rightarrow S^{3}(U)$ can be constructed as follows: Picking a basis in $V^{*}$ we can identify a net with a triple $M=\left(M_{1}, M_{2}, M_{3}\right)$ where $M_{i} \in S^{2} U^{*}$. We may think of $S^{3}(U)$ as the space of cubic polynomial functions on $U^{*}$. Now $\pi_{2}\left(M_{1} \wedge M_{2} \wedge M_{3}\right)$ vanishes on a linear form $f \in U^{*}$ if the net $M$ restricted to the zero locus of $f$ does not have full rank.

More explicitly, eliminate the variable $\nu$ from the ternary quadratic forms $M_{i}$ using the relation $x \xi+y \eta+z \nu=0$; we obtain three binary quadratic forms in the variables $\xi, \eta$. Now $\pi_{2}\left(M_{1} \wedge M_{2} \wedge M_{3}\right)$ is the determinant of the $3 \times 3$ matrix whose columns contain the coefficients of these three binary quadratic forms. In particular, $\pi_{2}\left(e_{i j k}\right)$ is the $(i, j, k)$ minor of

$$
\left(\begin{array}{cccccc}
z & 0 & -x & 0 & 0 & x^{2} / z \\
0 & 0 & 0 & z & -y & y^{2} / z \\
0 & z & -y & 0 & -x & 2 x y / z
\end{array}\right)
$$

(showing also that we end up with a cubic polynomial in $x, y, z$ ). The dual $\pi_{2}^{*}$ embeds $S^{3} U^{*}$ into $\mathbf{P l}^{*}$, and in the same way as in the case of the Jacobi map one may check that the basis vectors $\xi^{3}, \xi^{2} \eta, \ldots, \nu^{3}$ are mapped to $\frac{-1}{3} w_{1}^{*}, \ldots, \frac{-1}{3} w_{10}^{*}$ from Table 2 .

5.5. Stability. A net of conics is in the nullcone if both $J_{6}$ and $J_{12}$ are zero on it. The G.I.T. quotient of Noc is $\mathbf{P}^{1}$ and the quotient map on the complement of the nullcone is given by $k:=J_{6}^{2} / J_{12}$. An orbit $\eta$ is strictly semistable if $k^{-1}(k(\eta)) \supsetneqq \eta$. We can use formula (6) to calculate $J_{6}$ and the explicit form of the degree 4 invariant of the plane cubics to calculate $J_{12}$. 
Notice that Theorem 5.1 is not sufficient, since non-smooth cubics do not admit a Weierstrass form. Nevertheless these are simple calculations which show that the only codimension $>1$ orbits outside the nullcone are $D, D^{*}, E, E^{*}$ with

$\begin{array}{cccc} & J_{6} & J_{12} & k \\ D & 1 & 1 & 1 \\ D^{*} & -8 & 16 & 4 \\ E & 1 & 1 & 1 \\ E^{*} & -8 & 16 & 4\end{array}$

Since $k$ is a bijection on the codimension 1 orbits it is enough to find values of $c$ and $g$ with the given $k$. Since $k\left(\nu_{c, g}\right)=\frac{12 g^{2}}{3 g^{2}-c}$, it is immediate that $k(B)=1$ for $B:=\nu_{-9,1}$ and $k\left(B^{*}\right)=4$ for $B^{*}:=\nu_{0,1}\left(B\right.$ and $B^{*}$ are notations from [Wal77]). Consequently the complete list of strictly semistable orbits are $B, B^{*}, D, D^{*}, E, E^{*}$.

5.5.1. The discriminant. For the representations Noc and $S^{3} \mathbb{C}^{3}$ the nonstable variety is a hypersurface and we call their defining equation the discriminant of the representation. It is a classical result that the discriminant of the plane cubics is $\Delta=4 a^{3}+27 b^{2}$. Using the $c$ - $g$-form we can quickly check that $-2^{8} 3^{3} \delta^{*} \Delta=\left(J_{6}^{2}-J_{12}\right)^{2}\left(J_{6}^{2}-4 J_{12}\right)$ (see Section 7.1 for the details), consequently a net is unstable if and only if its determinant cubic is unstable, but the $\delta^{*}$-image of the discriminant is not the discriminant: the component $\left(J_{6}^{2}-J_{12}\right)=\bar{B}$ is counted with multiplicity 2 .

\section{Hierarchy of the Nets of CONICS}

C. T. C. Wall's result on the classification of the Noc-orbits can be verified using the results of Sections 3 and 5 . The codimension 1 orbits are classified by their $k$-invariant. The fact stated in Theorem 3.1, namely that the restriction equations determine the equivariant classes imply that no orbit is missed in Table 1. Any missing orbit would cause an indeterminacy in the solution of the restriction equations, hence would contradict to Theorem 3.1.

To determine the hierarchy we use that a cohomologically defined incidence class determines adjacency for positive orbits: Consider a Lie group $G$ acting on a vector space $V$ complex linearly. For $v \in V$ let $T_{v}$ denote the maximal torus of the stabilizer subgroup of $G$.

Definition 6.1. The orbit $G v$ is positive if there is a linear functional $\varphi$ on the weight lattice of $T_{v}$ such that for all weights $w_{i}$ of the $T_{v}$-action on the normal space of the orbit $G v$ at $v$ we have $\varphi\left(w_{i}\right)>0$.

Theorem 6.2. [FP09] Let $\eta \subset V$ be a $G$-invariant subvariety and suppose that the orbit $G v$ is positive for some $v \in V$. Then $v \in \eta$ if and only if $[\eta \subset V]_{T_{v}} \neq 0$.

Table 3 contains the description of normal slices to orbits, and their weights. The last column contains the values of the functional $\varphi$ (that is, the values $\varphi(\alpha), \varphi(\beta), \ldots$ ) if such a functional, proving the positivity of the given orbit, exists. By inspection we obtain the following fact.

Proposition 6.3. All unstable orbits of Noc are positive.

Thus, Theorem 6.2 determines almost all adjacencies of the orbits, namely the ones involving unstable orbits. The missing adjacencies of the semistable orbits can be determined by calculating the $k$-invariant. As a result we obtain the complete hierarchy, depicted on Figure 2.

Example 6.4. Consider the orbits $F$ and $F^{*}$, and their adjacency with the orbit $\left(1^{4}\right)$. Let $v$ be the point in the $\left(1^{4}\right)$ orbit given in the Table, and let $j_{\left(1^{4}\right)}$ be the restriction homomorphism 
TABLE 3. Normal weights and positivity

\begin{tabular}{|c|c|c|c|c|}
\hline$\Sigma^{0}$ & & & normal weights & $\varphi(\alpha), \varphi(\beta), \ldots$ \\
\hline$C$ & 2 & $y^{2}+2 x z, 2 y z,-x^{2}$ & $4 \alpha-4 \beta, 2 \alpha-2 \beta$ & 1,0 \\
\hline$D$ & 2 & $x^{2}, y^{2}, z^{2}+2 x y$ & $3 \alpha-3 \beta, 3 \beta-3 \alpha$ & - \\
\hline$D^{*}$ & 2 & $2 x z, 2 y z, z^{2}+2 x y$ & $3 \alpha-3 \beta, 3 \beta-3 \alpha$ & - \\
\hline$E$ & 3 & $x^{2}, y^{2}, z^{2}$ & $2 \alpha-\beta-\gamma, 2 \beta-\alpha-\gamma, 2 \gamma-\alpha-\beta$ & - \\
\hline$E^{*}$ & 3 & $2 x y, 2 y z, 2 z x$ & $-2 \alpha+\beta+\gamma,-2 \beta+\alpha+\gamma,-2 \gamma+\alpha+\beta$ & - \\
\hline$F$ & 3 & $x^{2}+y^{2}, 2 x y, 2 y z$ & $2 \alpha-2 \beta, 2 \alpha-2 \beta, \alpha-\beta$ & 1,0 \\
\hline$F^{*}$ & 3 & $x^{2}+y^{2}, x z, z^{2}$ & $2 \beta-2 \alpha, 2 \beta-2 \alpha, \beta-\alpha$ & $-1,0$ \\
\hline G & 4 & $x^{2}, y^{2}, y z$ & $2 \alpha-2 \gamma, \beta-\alpha, 2 \beta-\alpha-\gamma, 2 \beta-2 \gamma$ & $1,2,0$ \\
\hline$G^{*}$ & 4 & $x y, x z, z^{2}$ & $2 \gamma-2 \alpha, \alpha-\beta,-2 \beta+\alpha+\gamma, 2 \gamma-2 \beta$ & $-1,-2,0$ \\
\hline$H$ & 5 & $x^{2}, 2 x y, y^{2}+2 x z$ & $2 \alpha-2 \beta, 2 \alpha-2 \beta, 3 \alpha-3 \beta, 3 \alpha-3 \beta, 4 \alpha-4 \beta$ & 1,0 \\
\hline$I$ & 7 & $x^{2}, x y, y^{2}$ & $\alpha-\gamma, 2 \alpha-\beta-\gamma, 2 \alpha-2 \gamma, \beta-\gamma,-2 \gamma+\alpha+\beta, 2 \beta-2 \gamma$ & $0,0,-1$ \\
\hline$I^{*}$ & 7 & $x z, y z, z^{2}$ & $-\alpha+\gamma,-2 \alpha+\beta+\gamma,-2 \alpha+2 \gamma,-\beta+\gamma, 2 \gamma-\alpha-\beta,-2 \beta+2 \gamma$ & $0,0,1$ \\
\hline \multicolumn{5}{|c|}{ ( } \\
\hline$\left(1^{4}\right)$ & 4 & $x^{2}-x z, y^{2}-y z, 0$ & $\beta-2 \gamma, \beta-2 \gamma, \beta-2 \gamma, \beta-2 \gamma$ & 1,0 \\
\hline$\left(21^{2}\right)$ & 5 & $x y, x z+y z, 0$ & $2 \alpha-2 \beta, \gamma-2 \alpha, \gamma-2 \alpha, \gamma-\alpha-\beta, \gamma-2 \beta$ & $1,0,3$ \\
\hline$(31)$ & 6 & $x z, x^{2}-y z, 0$ & $3 \beta-3 \alpha, 2 \beta-2 \alpha, \gamma-3 \alpha-\beta, \gamma-4 \alpha, \gamma-2 \alpha-2 \beta, \gamma-4 \beta$ & $0,1,5$ \\
\hline$(22)$ & 6 & $x^{2}, y z, 0$ & $2 \alpha-2 \beta, 2 \alpha-2 \gamma, \delta-\alpha-\beta, \delta-\alpha-\gamma, \delta-2 \beta, \delta-2 \gamma$ & $1,0,0,2$ \\
\hline$(4)$ & 7 & $x z+y^{2}, x^{2}, 0$ & $2 \alpha-2 \beta, 3 \alpha-3 \beta, 4 \alpha-4 \beta, \gamma-3 \alpha-\beta, \gamma-2 \alpha-2 \beta, \gamma-4 \beta$ & $1,0,5$ \\
\hline$K$ & 8 & $y^{2}, z^{2}, 0$ & $\begin{array}{l}2 \alpha-2 \beta, 2 \alpha-\beta-\gamma, 2 \gamma-2 \beta, 2 \gamma-\alpha-\beta, \delta-2 \beta \\
\delta-\alpha-\beta, \delta-\beta-\gamma, \delta-\alpha-\gamma\end{array}$ & $0,-1,0,0$ \\
\hline$L$ & 8 & $x y, x z, 0$ & $\alpha-\beta, \alpha-\gamma, \alpha+\beta-2 \gamma, \alpha+\gamma-2 \beta, \delta-2 \alpha, \delta-2 \beta$ & \\
\hline$M$ & 9 & & $\begin{array}{l}\delta-2 \gamma, \delta-\beta-\gamma \\
\beta+\gamma-2 \alpha, \beta-\alpha, 2 \beta-2 \alpha, 2 \beta-\alpha-\gamma, 2 \beta-2 \gamma, \delta-2 \alpha\end{array}$ & $1,0,0,3$ \\
\hline 101 & J & $90,9,0$ & $\delta-\alpha-\beta, \delta-\alpha-\gamma, \delta-2 \gamma$ & $0,1,0,2$ \\
\hline \multicolumn{5}{|c|}{ e } \\
\hline$S$ & 10 & $x y-z^{2}, 0,0$ & $\begin{array}{l}\gamma-2 \alpha-2 \beta, \gamma-3 \alpha-\beta, \gamma-4 \alpha, \gamma-\alpha-3 \beta, \gamma-4 \beta \\
\delta-2 \alpha-2 \beta, \delta-3 \alpha-\beta, \delta-4 \alpha, \delta-\alpha-3 \beta, \delta-4 \beta\end{array}$ & $0,0,1,1$ \\
\hline$P L$ & 11 & $x y, 0,0$ & $\begin{array}{l}\delta-2 \alpha, \delta-2 \beta, \delta-\alpha-\gamma, \delta-\beta-\gamma, \delta-2 \gamma \\
\epsilon-2 \alpha, \epsilon-2 \beta, \epsilon-\alpha-\gamma, \epsilon-\beta-\gamma, \epsilon-2 \gamma, \alpha+\beta-2 \gamma\end{array}$ & $1,0,0,3,3$ \\
\hline$D L$ & 13 & $x^{2}, 0,0$ & $\begin{array}{l}\delta-\alpha-\beta, \delta-2 \beta, \delta-\alpha-\gamma, \delta-2 \gamma, \delta-\beta-\gamma \\
\epsilon-\alpha-\beta, \epsilon-2 \beta, \epsilon-\alpha-\gamma, \epsilon-2 \gamma, \epsilon-\beta-\gamma \\
2 \alpha-2 \beta, 2 \alpha-\beta-\gamma, 2 \alpha-2 \gamma\end{array}$ & $1,0,0,3,3$ \\
\hline
\end{tabular}

$H_{\mathrm{GL}(U) \times \mathrm{GL}(V)}^{*}(\mathbf{N o c}) \rightarrow H_{T_{v}}^{*}(\mathbf{N o c})$. One can read from the table above that the homomorphism $j_{\left(1^{4}\right)}$ is the substitution

$$
c_{i}=\sigma_{i}(\alpha, \alpha, \alpha), \quad d_{i}=\sigma_{i}(2 \alpha, 2 \alpha, \beta),
$$

where $\sigma_{i}$ denotes the $i$ th elementary symmetric polynomial. For the equivariant classes given in Theorem 3.1 we have

$$
j_{\left(1^{4}\right)}([\bar{F}])=0, \quad j_{\left(1^{4}\right)}\left(\left[\overline{F^{*}}\right]\right)=-6(2 \alpha-\beta)\left(4 \alpha^{2}-4 \alpha \beta+\beta^{2}\right) \neq 0 .
$$

Hence, we have that $\left(1^{4}\right)$ is contained in the orbit closure of $F^{*}$, but is not contained in the orbit closure of $F$.

6.1. The equivariant cohomology rings of the orbits. With a little extra sudoku type calculations one can determine the equivariant cohomology rings $H^{*}\left(B G_{x}\right)$ of the orbits $G x$. In column 5 of Table 1 we listed the degrees of a free generating set for these rings. This information can be used, e.g., to define certain "higher" Thom polynomials. Since the equivariant cohomology spectral sequence of the codimension filtration (the Kazarian spectral sequence; see 


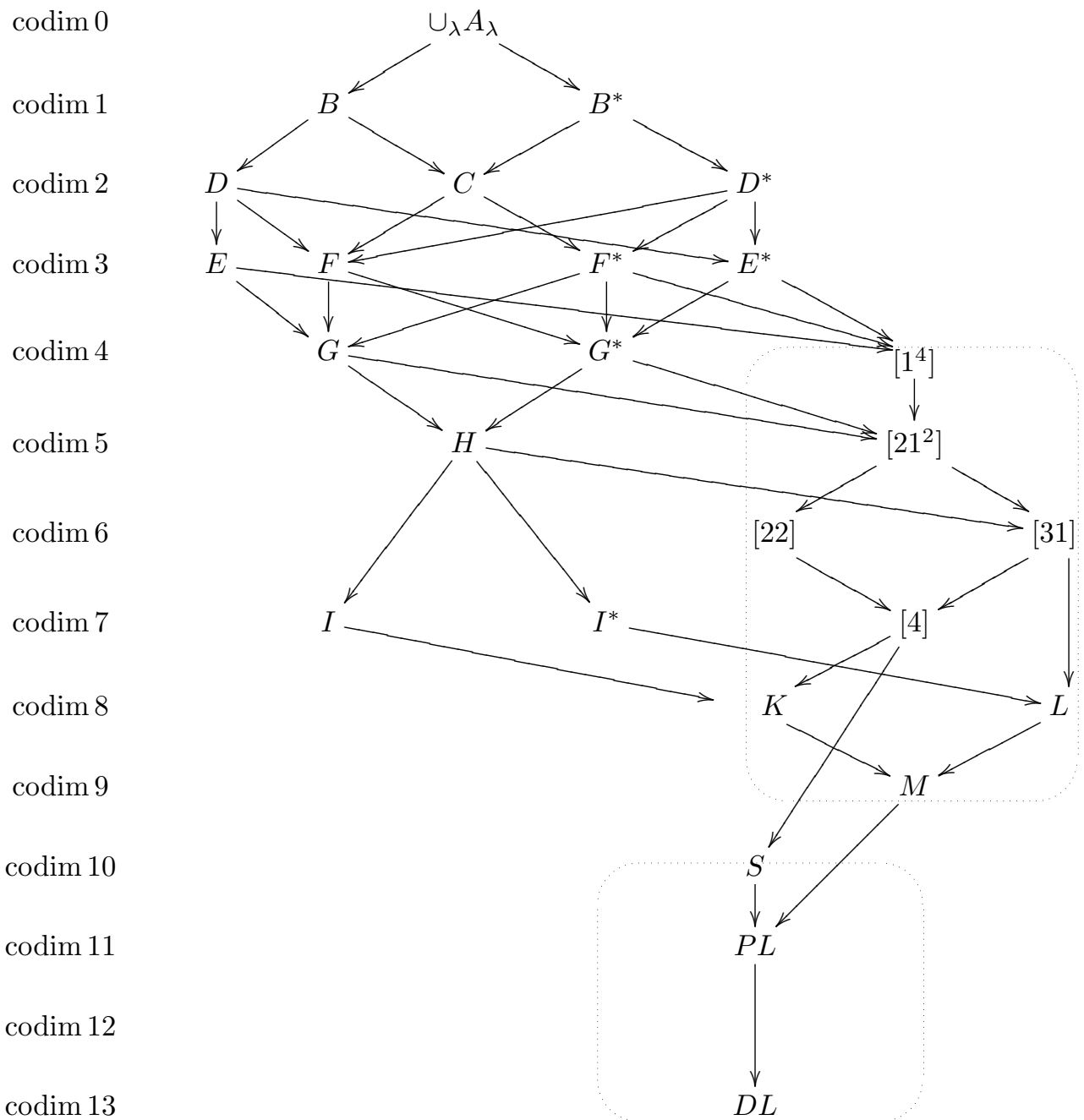

Figure 2. The hierarchy of Noc orbits

[Kaz97], [FR04, Sect.10]) degenerates, the Poincaré series of the rings $H^{*}\left(B G_{x}\right)$ shifted by the codimension add up to the Poincare series of $H^{*}(B G)$. For the open stratum $O=\bigcup_{\mu \in \mathbf{P}^{1}} A_{\mu}$ we have $H_{G}^{*}(O)=H_{\mathrm{GL}_{1}}^{*}\left(\mathbf{P}^{1}\right)$ for the trivial $\mathrm{GL}_{1}$-action, so the Poincaré series of $H_{G}^{*}(O)$ is $\frac{1+t}{1-t}$, and we get that

$\frac{1+t}{1-t}+\frac{t^{2}}{(1-t)^{2}}+\frac{2 t^{2}}{(1-t)\left(1-t^{2}\right)}+\ldots+\frac{t^{18}}{(1-t)^{2}\left(1-t^{2}\right)^{2}\left(1-t^{3}\right)^{2}}=\frac{1}{(1-t)^{2}\left(1-t^{2}\right)^{2}\left(1-t^{3}\right)^{2}}$.

Here the $t$-exponents of the numerator are the codimensions (column 2) and the $t$-exponents of the denominators are degrees of the generators of $H^{*}\left(B G_{x}\right)$ (column 5). 


\section{Enumerative QUestions, MUltiplicities of THE DETERMinAnT MAP}

Equivariant classes contain enumerative data in a compressed form. One of the simplest enumerative invariants is the degree. Using [FNR05, Section 6] we see that the degree of a $G$ invariant subvariety $\eta \subset$ Noc can be obtained by substituting 1 to the Chern roots of GL $(V)$ and 0 to the Chern roots of $\mathrm{GL}(U)$ in the polynomial $[\eta] \in H^{*}(B(\mathrm{GL}(U) \times \mathrm{GL}(V)))$. For example, we obtain

$$
\operatorname{deg}(\bar{C})=72, \quad \operatorname{deg}(\bar{D})=36, \quad \operatorname{deg}\left(\overline{D^{*}}\right)=45 .
$$

Consequently in a generic two-parameter linear family of nets of conics there are 72 of type $C$, 36 of type $D$ and 45 of type $D^{*}$, etc.

A more subtle enumerative invariant is the intersection multiplicity. Suppose that $f: X \rightarrow$ $Y$ is a map of smooth varieties and $Z \subset Y$ a codimension $d$ subvariety. Suppose also that the preimage $f^{-1} Z$ is pure $d$-codimensional. Then we can assign positive integers $\mu_{i}$ to every component $Y_{i}$ of $f^{-1} Z$ called intersection multiplicities (for more details see [Ful84]). If $X=$ $Y=\mathbb{C}$ and $Z=\{0\}$, then the intersection multiplicities are the usual multiplicities of the roots of $f$.

An important property of the intersection multiplicity ([Ful84, Sect. 7]) is that if all components $Y_{i}$ of $f^{-1} Z$ are of codimension $d$, then

$$
f^{*}([X])=\sum \mu_{i}\left[Y_{i}\right] .
$$

In general this equation does not determine the intersection multiplicities $\mu_{i}$. However (7) generalizes to the equivariant setting where there is more chance that the classes $\left[Y_{i}\right]$ will be linearly independent.

Consider the determinant map $\delta:$ Noc $\rightarrow S^{3} V$ studied in Section 5.2. From the normal forms in Table 1 it is easy to calculate the image under the determinant map. E.g., for $C$ the normal form is $y^{2}+2 x z, 2 y z,-x^{2}$ which means that the generic net in matrix form is

$$
\left(\begin{array}{ccc}
-\kappa & \cdot & \lambda \\
\cdot & \lambda & \mu \\
\lambda & \mu & \cdot
\end{array}\right),
$$

with determinant $\mu^{2} \kappa-\lambda^{3}$ corresponding to the cuspidal curve $\nu$. A table can be found in [Wal77]. For the readers' convenience we included the images of the determinant map in the last column of Table 1 . The notation tries to imitate the shape of the various classes of plane cubics, i. e. $\theta$ is the orbit (closure) of conic intersected by line, $\Omega$ the conic and tangent line, A the three nonconcurrent lines, $\neq$ is the double line intersected by a third line, $\Xi$ is the triple line and $K$ is the three concurrent lines. The codimension 1 orbits will be treated in the next section.

The equivariant classes of the $\mathrm{GL}_{3}$-representation $S^{3} \mathbb{C}^{3}$ (i.e., plane cubics) were calculated by B. Kömüves [Köm03]. The cases we need are

(8) $[\nu]=24 e_{1}^{2},[\theta]=18 e_{1}^{2}+9 e_{2},[\Omega]=36 e_{1}^{3}+18 e_{1} e_{2},[\mathrm{~A}]=12 e_{1}^{3}+6 e_{1} e_{2}+27 e_{3},[\mathrm{~K}]=e_{1}[\mathrm{~A}]$,

where $e_{i}$ denote the $\mathrm{GL}_{3}$-Chern classes.

The map $\delta$ is $\mathrm{GL}_{3} \times \mathrm{GL}_{3}$-equivariant, if we replace the $\mathrm{GL}_{3}$-action on the plane cubics by the $\mathrm{GL}(U) \times \mathrm{GL}(V)$-action as in Section 5.2. The effect of this change on (8) is replacing the $\mathrm{GL}_{3}$-Chern roots $\epsilon_{i}\left(\epsilon_{1}+\epsilon_{2}+\epsilon_{3}=e_{1}\right.$ etc) by some linear combination of the $\mathrm{GL}(U) \times \mathrm{GL}(V)$ Chern roots. A comparison of the actions of the maximal tori of $\mathrm{GL}_{3}$ and $\mathrm{GL}(U) \times G L(V)$ gives the substitution $\epsilon_{i} \mapsto \delta_{i}-2 / 3 u_{1}$ (where $\delta_{i}$ denote the $\mathrm{GL}(V)$-Chern roots). Consequently for the Chern classes we obtain the substitution $e_{1} \mapsto v_{1}-2 u_{1}, e_{2} \mapsto v_{2}-4 / 3 u_{1} v_{1}+4 / 3 u_{1}^{2}$ and $e_{3} \mapsto v_{3}-2 / 3 v_{2} u_{1}+4 / 9 v_{1} u_{1}^{2}-8 / 27 u_{1}^{3}$. 
After this substitution we have all the ingredients of (7) for the $\mathrm{GL}(U) \times \mathrm{GL}(V)$-equivariant map $\delta$. Then explicit calculation implies the following theorem.

Theorem 7.1. In $\mathrm{GL}(U) \times \mathrm{GL}(V)$-equivariant cohomology we have

$$
\begin{gathered}
\delta^{*}([\nu])=3[C], \\
\delta^{*}([\theta])=4[D]+\left[D^{*}\right], \\
\delta^{*}([\Omega])=9[F]+6\left[F^{*}\right], \\
\delta^{*}([\mathrm{~A}])=8[E]+\left[E^{*}\right]+2[F],
\end{gathered}
$$

and the coefficients on the right hand side are uniquely determined, therefore they are the intersection multiplicities.

For the three concurrent lines the codimension condition is not satisfied, but a similar calculation gives the equality

$$
\delta^{*}([K])=12\left[\left(1^{4}\right)\right]+\left(4[G]+1 / 2\left[G^{*}\right]+2\left(d_{1}-2 c_{1}\right)[F]\right),
$$

and, like above, the coefficients are uniquely determined. The class in the bracket is supported on $\bar{F}$, so we can call 12 the intersection multiplicity of $\left(1^{4}\right)$.

Remark 7.2. The intersection multiplicities are always at most the algebraic multiplicities by [Ful84, Pr. 8.2] and they agree if the image is (locally) Cohen-Macaulay and the preimage has the same codimension by [FP98, p.108]. In [Chi02] J. Chipalkatti determines which orbit closures are arithmetically Cohen-Macaulay for the plane cubics. Among the orbits of Theorem 7.1 $\nu$ is Cohen-Macaulay, since it is a complete intersection, $\Omega$ is arithmetically Cohen-Macaulay consequently Cohen-Macaulay, but $\theta$ and $\mathrm{A}$ are not arithmetically Cohen-Macaulay. Therefore the intersection multiplicities for $\nu$ and $\Omega$ are algebraic multiplicities as well. For $\theta$ and A we do not know if they are Cohen-Macaulay. If the algebraic multiplicities for $\theta$ and A differ from the intersection multiplicities, then they cannot be Cohen-Macaulay. Unfortunately we were not able to calculate these algebraic multiplicities.

For the codimension 1 orbits we study the induced map on the GIT quotients.

7.1. The induced map on the GIT quotients of nets of conics to plane cubics. To see that the $G=\mathrm{GL}(U) \times \mathrm{GL}(V)$-equivariant determinant map $\delta$ induces a map $d$ of the corresponding GIT quotients we need to check that semistable orbits are mapped to semistable orbits. It follows from general principles but here is a direct verification. For codimension $>1$ orbits of Noc we see this fact from Table 1. (For the plane cubics the semistable orbits are the smooth curves which are also stable, together with the nodal curve and $\theta$ and A.) The codimension 1 orbits $A_{\mu}$ have a $\nu_{c, g}$ representative with $(c, g) \neq(0,0)$, so for $\delta\left(\nu_{c, g}\right)$ either $a=c-3 g^{2}$ or $b=2 g\left(c+g^{2}\right)$ is not zero, therefore the image is semistable.

The traditional parametrization of the GIT quotient $S^{3} \mathbb{C}^{3} / / G$ is the $j$-invariant $j=\frac{4 a^{3}}{\Delta}$ : $S^{3} \mathbb{C}^{3} / / G \rightarrow \mathbf{P}^{1}$, where $\Delta=4 a^{3}+27 b^{2}$ is the discriminant. On the other hand we saw in Section 5.5 that the invariant $k=\frac{J_{6}^{2}}{J_{12}}:$ Noc $/ / G \rightarrow \mathbf{P}^{1}$ parametrizes the GIT quotient Noc $/ / G$. Using these parametrizations we can calculate $\tilde{d}:=j \circ d \circ k^{-1}: \mathbf{P}^{1} \rightarrow \mathbf{P}^{1}$ :

Using the $c-g$-form we get (by some abuse of notation)

$$
\Delta=\Delta\left(\nu_{c, g}\right)=4 a^{3}+27 b^{2}=4 a^{3}+27\left(4 g^{2}\right)\left(a+4 g^{2}\right)^{2}=4\left(a+3 g^{2}\right)\left(a+12 g^{2}\right)^{2},
$$

and we have $J_{6}=24 g$ and $J_{12}=-48 a$, so $48^{3} \Delta=\left(-4 J_{12}+J_{6}^{2}\right)\left(-J_{12}+J_{6}^{2}\right)^{2}$. Therefore $j \circ d=\frac{J_{12}^{3}}{\left(J_{6}^{2}-4 J_{12}\right)\left(J_{6}^{2}-J_{12}\right)^{2}}$ and

$$
\tilde{d}(x: y)=\left(4 y^{3}:(x-4 y)(x-y)^{2}\right) .
$$


The branching points are at the singular points (take the affine chart $y=1$, and find the zeroes of $3(x-1)(x-3)$, the derivative of $(x-4)(x-1)^{2}$, and an extra branching at $x=\infty$.):

$x=1(\Delta=0$, i.e., $j$-invariant is $\infty)$ corresponds to the semistable point (class of the nodal curve). It has preimages $B$ with $k(B)=1$ and $B^{*}$ with $k\left(B^{*}\right)=4$. The point $B$ has multiplicity two. Notice that $B$ and $B^{*}$ represent the two semistable points in Noc $/ / G$ (in the GIT quotient $B \sim D \sim E$ and $B^{*} \sim D^{*} \sim E^{*}$ ).

$x=\infty$ ( $j$-invariant is 0$)$ corresponds to the degree 4 orbit of elliptic curves defined by $a=0$. It has one preimage (of multiplicity 3 ): the orbit of nets of conics defined by $J_{12}=0$.

$x=3$ ( $j$-invariant is 1 ) corresponds to the degree 6 orbit of elliptic curves defined by $b=0$. It has one preimage with $k=3$ and another one with multiplicity 2, the degree 6 orbit of nets of conics defined by $J_{6}=0$.

Remark 7.3. For hypersurfaces the intersection multiplicities agree with the algebraic (or scheme theoretic) multiplicities. To determine these multiplicities in our case it is enough to compare degrees. One obtains that all algebraic multiplicities are 1, except for $B$ which has multiplicity 2 .

\section{REFERENCES}

[AN02] D. I. Artamkin and A. G. Nurmiev. Orbits and invariants of third-order cubic matrices with symmetric fibers. Mathematical Notes, 72(4):447-453, 2002. DOI: 10.1023/A:1020572026013.

[Aro50] S. Aronhold. Zur theorie der homogenen functionen dritten grades von drei variablen. Crelle J., 39:140159, 1850. DOI: $10.1515 /$ crll.1850.39.140.

[BLT04] E. Briand, J-G. Luque, and J-Y. Thibon. The moduli space of three-qutrit states. J. Math. Phys., 45:4855-4867, 2004. DOI: 10.1063/1.1809255.

[BS12] Gergely Bérczi and András Szenes. Thom polynomials of Morin singularities. Ann. of Math., 175(2):567629, 2012. DOI: 10.4007/annals.2012.175.2.4.

[Chi02] Jaydeep V. Chipalkatti. Decomposable ternary cubics. Experimental Mathematics, 11(1):69-80, 2002. DOI: $10.1080 / 10586458.2002 .10504469$.

[DD12] M. Domokos and V. Drensky. Defining relation for semi-invariants of three by three matrix triples. $J$. Pure Appl. Alg, 216:2098-2105, 2012. DOI: 10.1016/j.jpaa.2012.01.017.

[FNR05] L. M. Fehér, A. Némethi, and R. Rimányi. Degeneracy of two and three forms. Canad. Math. Bull., 48(4):547-560, 2005.

[FP98] W. Fulton and P. Pragacz. Schubert varieties and degeneracy loci. Number 1689 in Lecture Notes in Mathematics. Springer-Verlag, 1998.

[FP09] László M. Fehér and Zsolt Patakfalvi. The incidence class and the hierarchy of orbits. Cent. European J. Math., 7(3):429-441, September 2009. DOI: 10.2478/s11533-009-0026-3.

[FR04] L. M. Fehér and R. Rimányi. Calculation of Thom polynomials and other cohomological obstructions for group actions. In Real and Complex Singularities (Sao Carlos, 2002), number 354 in Contemp. Math., pages 69-93. Amer. Math. Soc., 2004.

[FR12] L. M. Fehér and R. Rimányi. Thom series of contact singularities. Ann. of Math., 176(3):1381-1426, November 2012. DOI: 10.4007/annals.2012.176.3.1.

[Ful84] William Fulton. Intersection Theory. Number 3. Folge Band 2 in Modern Surveys in Mathematics. Springer-Verlag, 1984.

[Giz07] M. Gizatullin. On covariants of plane quartic associated to its even theta characteristic. In Algebraic geometry: Korea-Japan Conference in honor of Igor Dolgachev, Volume 10, Contemp. Math., 422, pages 37-74. Amer. Math. Soc., 2007.

[Kaz] M. É. Kazarian. Non-associative Hilbert scheme and Thom polynomials. Unpublished.

[Kaz97] M. É. Kazarian. Characteristic classes of singularity theory. In The Arnold-Gelfand mathematical seminars, pages 325-340. Birkhäuser Boston, 1997.

[Kőm03] Balázs Kômúves. Thom polynomials via restriction equations. 2003. Undergraduate Thesis.

[Mac98] I. G. Macdonald. Symmetric functions orthogonal polynomials, volume 12 of University Lectures Series, Rutgers. AMS, 1998.

[Mat71] J. N. Mather. Stability of $C^{\infty}$ mappings VI: the nice dimensions. In C. T. C. Wall, editor, Proceedings of Liverpool Singularities I, LNM 192. Springer, 1971. DOI: 10.1007/BFb0066824. 
[Mul09] Ketan D. Mulmuley. On P vs. NP, geometric complexity theory, and the Riemann hypothesis. ar $\chi$ iv: 0908.1936, 2009.

[Pro07] C. Procesi. Lie groups, An approach through invariants and representations. Springer,London, 2007.

[Rim01] R. Rimányi. Thom polynomials, symmetries and incidences of singularities. Inv. Math., 143:499-521, 2001.

[Sal79] G. Salmon. A treatise on conic sections. Longmans, Green, and Co., 1879.

[Vin76] E. B. Vinberg. The Weyl group of a graded lie algebra (Russian). Izv. Akad. Nauk SSSR Ser. Mat., 40(3):488-526, 1976.

[Wal77] C. T. C. Wall. Nets of conics. Math. Proc. Cambridge Philos. Soc., 81(3):351-364, 1977. DOI: $10.1017 /$ S0305004100053421.

[Wal10] C. T. C. Wall. Note on the invariant of nets of conics. 2010.

[WdP95] C. T. C. Wall and A. A. du Plessis. The geometry of topological stability. Number 9 in London Math. Soc. Monographs, new series. Oxford University Press, 1995.

Rényi Institute of Mathematics, Hungarian Academy of Sciences, Budapest, Hungary

E-mail address: domokos.matyas@renyi.mta.hu

Department of Analysis, Eotvos University, Budapest, Hungary

E-mail address: lfeher@renyi.mta.hu

Department of Mathematics, University of North Carolina, Chapel Hill, NC, USA

E-mail address: rimanyi@email.unc.edu 